TRANSACTIONS OF THE

AMERICAN MATHEMATICAL SOCIETY

Volume 355, Number 3, Pages 1269-1294

S 0002-9947(02)03169-0

Article electronically published on November 5, 2002

\title{
HYPERBOLIC MEAN GROWTH OF BOUNDED HOLOMORPHIC FUNCTIONS IN THE BALL
}

\author{
E. G. KWON
}

\begin{abstract}
We consider the hyperbolic Hardy class $\varrho H^{p}(B), 0<p<\infty$. It consists of $\phi$ holomorphic in the unit complex ball $B$ for which $|\phi|<1$ and

$$
\sup _{0<r<1} \int_{\partial B}\{\varrho(\phi(r \zeta), 0)\}^{p} d \sigma(\zeta)<\infty
$$

where $\varrho$ denotes the hyperbolic distance of the unit disc. The hyperbolic version of the Littlewood-Paley type $g$-function and the area function are defined in terms of the invariant gradient of $B$, and membership of $\varrho H^{p}(B)$ is expressed by the $L^{p}$ property of the functions. As an application, we can characterize the boundedness and the compactness of the composition operator $\mathcal{C}_{\phi}$, defined by $\mathcal{C}_{\phi} f=f \circ \phi$, from the Bloch space into the Hardy space $H^{p}(B)$.
\end{abstract}

\section{INTRODUCTION}

1.1. Let $\phi$ be a holomorphic map from the unit ball $B=B_{n}$ of $\mathbb{C}^{n}$ into the unit disc of $\mathbb{C}$ and let $\mathcal{C}_{\phi}$ be the composition operator defined by $\mathcal{C}_{\phi} f=f \circ \phi$. Then properties of $\phi$ can be observed either in terms of the hyperbolic growth of the image $\phi(r B)$ in $B_{1}$ or by the operator-theoretic behavior of $\mathcal{C}_{\phi}$. Moreover, there should be a relationship between the hyperbolic growth of $\phi$ and certain properties of $\mathcal{C}_{\phi}$. For example, when $n=1, \phi$ is hyperbolically $\mathcal{B}_{0}$ in the sense of $\lim _{|z| \rightarrow 1}\left(1-|z|^{2}\right) \frac{\left|\phi^{\prime}(z)\right|}{1-|\phi(z)|^{2}}=0$ if and only if $\mathcal{C}_{\phi}$ is compact on the little Bloch space $\mathcal{B}_{0}([\mathrm{CM}$, p. 194]).

From the viewpoint of the boundary behavior, there was a branch of the study of $\mathcal{C}_{\phi}$ from the Bloch space $\mathcal{B}$ into a nice function space. P. Ahern ([A]) observed that $C_{\phi} g \in B M O A(B)$ for all $g \in \mathcal{B}$ and for a monomial $\phi$. Extensive research followed. Several examples of homogeneous polynomials and conditions for $\phi$ were found to have the same property and, finally, the relation with hyperbolic mean growth occurred. See [A], AR1], AR2], [C1], C22, [CC], CK], CRU, [K5], [Ma], [RU], Rus and [Sta]. The boundedness of $\mathcal{C}_{\phi}: \mathcal{B} \rightarrow B M O A$ can be characterized by $\phi \in \varrho B M O A$, where $\varrho B M O A$ is the hyperbolic $B M O A$ class ( $\underline{\mathrm{K}}$, see $\mathrm{Y} 3$ ) for $\left.\varrho B M O A\left(B_{1}\right)\right)$. Also, in the same vein, the boundedness of $\mathcal{C}_{\phi}: \mathcal{B} \rightarrow H^{2 p}\left(B_{1}\right)$ was characterized by $\phi \in \varrho H^{p}\left(B_{1}\right)$ when $1 \leq p<\infty$ ([K1]). Note that functions of the

Received by the editors May 15, 2001.

2000 Mathematics Subject Classification. Primary 30D45, 32A35, 47B33.

Key words and phrases. $H^{p}$ space, Bloch space, hyperbolic Hardy class, composition operator, Littlewood-Paley $g$-function, invariant gradient.

This work was supported by grant No. R01-2000-000-00001-0 from the Basic Research Program of the Korea Science \& Engineering Foundation. 
Hardy space $H^{p}$ and of $B M O A$ should have radial limits almost everywhere but Bloch functions need not.

1.2. In this paper we define and consider the hyperbolic Hardy class $\varrho H^{p}(B)$, $0<p<\infty$. It is defined to consist of holomorphic $\phi$ in $B$ for which $|\phi|<1$ and

$$
\sup _{0<r<1} \int_{\partial B} \varrho(\phi(r \zeta), 0)^{p} d \sigma(\zeta)<\infty,
$$

where $\varrho$ denotes the non-Euclidean hyperbolic distance of $B_{1}$ :

$$
\varrho(z, w)=\frac{1}{2} \log \frac{|1-\bar{z} w|+|z-w|}{|1-\bar{z} w|-|z-w|}, \quad z, w \in B_{1} .
$$

Our objective is to find a hyperbolic version of the area function and the $g$ function (of Littlewood-Paley type) which characterize the membership of $\varrho H^{p}(B)$. This leads us to characterize the boundedness and the compactness of the composition operator $\mathcal{C}_{\phi}: \mathcal{B} \rightarrow H^{p}(B)$ in terms of the growth of $\phi$, extending variable results to $n \geq 1$ and to $0<p<\infty$. The main result shows that the following are equivalent:

$$
\begin{aligned}
& \phi \in \varrho H^{p}(B) ; \\
& \int_{B}\left(1-|z|^{2}\right)^{n}\left(\log \frac{1}{1-|\phi(z)|^{2}}\right)^{p-1} \frac{|\widetilde{\nabla} \phi(z)|^{2}}{\left(1-|\phi(z)|^{2}\right)^{2}} d \tau(z)<\infty \text {; } \\
& \int_{0}^{1} \frac{|\widetilde{\nabla} \phi(r \zeta)|^{2}}{\left(1-|\phi(r \zeta)|^{2}\right)^{2}} \frac{d r}{1-r} \in L^{p}(\partial B) ; \\
& \int_{D_{\alpha}(\zeta)} \frac{|\widetilde{\nabla} \phi(z)|^{2}}{\left(1-|\phi(z)|^{2}\right)^{2}} d \tau(z) \in L^{p}(\partial B) ; \\
& \sup \left\{\log \frac{1}{1-|\phi(z)|^{2}}: z \in D_{\alpha}(\zeta)\right\} \in L^{p}(\partial B) \text {; } \\
& \mathcal{C}_{\phi}: \mathcal{B} \rightarrow H^{2 p}(B) \text { is bounded; } \\
& \mathcal{C}_{\phi}: \mathcal{B} \rightarrow H^{2 p}(B) \text { is compact. }
\end{aligned}
$$

Here $\widetilde{\nabla}$ is the $\mathcal{M}$-invariant gradient of $B, \tau$ and $\sigma$ are the $\mathcal{M}$-invariant volume measure on $B$ and the surface measure on $\partial B$ respectively, and $D_{\alpha}(\zeta)$ is the Koranyi approach region. These concepts will be explained in Section 2. See Theorem 3.6, Theorem 4.2, Theorem 5.1, Theorem 5.10, Theorem 6.1, and Theorem 6.2 for precise results of this paper.

1.3. After introducing some terminology in Section 2, we present and prove our main results in Section 5. Section 3 is the preparatory section dealing with properties of the invariant Laplacian, invariant gradient and Green's theorem. In Section 4, a characterization of $\mathcal{M}$-harmonic Hardy space by an invariant $g$-function will be given. In terms of tangential and radial gradients, more of the conditions equivalent to the membership of $\varrho H^{p}(B)$ will be given in Section 6. Section 7 will close this paper by posing a general principle on hyperbolic function classes related to composition operators (defined on the Bloch space) and by giving an example illustrating our result. 


\section{Preliminaries}

2.1. Let $B=B_{n}$ be the open unit ball of $\mathbb{C}^{n}$ and $S$ the boundary of $B$. The Hermitian inner product and the associated Euclidean norm of $\mathbb{C}^{n}$ are denoted by $\langle z, w\rangle=\sum_{j=1}^{n} z_{j} \bar{w}_{j}$ and $|z|=\sqrt{\langle z, z\rangle}$. The dot product on $\mathbb{C}^{n}=\mathbb{R}^{2 n}$ is denoted by $z \cdot w=\sum_{j=1}^{n}\left\{\operatorname{Re} z_{j} \operatorname{Re} w_{j}+\operatorname{Im} z_{j} \operatorname{Im} w_{j}\right\}, z, w \in \mathbb{C}^{n}$. The Möbius group, i.e., the group of biholomorphic self-maps of $B$, will be denoted by $\mathcal{M}$. Let $\varphi_{z}, z \in B$, denote the self-map of $B$ defined by

$$
\varphi_{z}(w)=\frac{z-\frac{\langle w, z\rangle}{\langle z, z\rangle} z-\sqrt{1-|z|^{2}}\left(w-\frac{\langle w, z\rangle}{\langle z, z\rangle} z\right)}{1-\langle w, z\rangle}, \quad w \in \bar{B},
$$

if $z \neq 0$ and $\varphi_{0}(w)=-w, w \in B$. It is known that $\mathcal{M}$ consists of functions of the form $U \varphi_{z}$, where $U$ denotes a unitary operator on $\mathbb{C}^{n}$.

2.2. We let $\mathcal{D}, \nabla$, and $\Delta$ denote respectively the complex gradient of $\mathbb{C}^{n}$, the real gradient of $\mathbb{C}^{n}=\mathbb{R}^{2 n}$ and the Laplacian of $\mathbb{C}^{n}$ :

$$
\begin{gathered}
\mathcal{D}=\left(D_{1}, D_{2}, \ldots, D_{n}\right), D_{j}=\frac{\partial}{\partial z_{j}}, j=1,2, \ldots, n, \\
\nabla=\left(\frac{\partial}{\partial x_{1}}, \frac{\partial}{\partial y_{1}}, \ldots, \frac{\partial}{\partial x_{n}}, \frac{\partial}{\partial y_{n}}\right), z_{j}=x_{j}+i y_{j}, j=1,2, \ldots, n, \\
\Delta=4 \sum_{j=1}^{n} D_{j} \bar{D}_{j} .
\end{gathered}
$$

The Laplace-Beltrami operator associated with the Bergman kernel of $B$ will be denoted by $\widetilde{\Delta}$ :

$$
\widetilde{\Delta} f(a)=4\left(1-|a|^{2}\right) \sum_{j, k=1}^{n}\left(\delta_{j, k}-a_{j} \bar{a}_{k}\right)\left(D_{j} \bar{D}_{k} f\right)(a), \quad a \in B, f \in C^{2}(B) .
$$

It is known ([H] or Sto1 Proposition 3.1]) that $\widetilde{\Delta}$ is $\mathcal{M}$-invariant: $\widetilde{\Delta}(f \circ \psi)=$ $(\widetilde{\Delta} f) \circ \psi$, for all $\psi \in \mathcal{M}$. In particular,

$$
\widetilde{\Delta} f(a)=\Delta\left(f \circ \varphi_{a}\right)(0), \quad a \in B,
$$

for $f \in C^{2}(B)$. In a similar way, we will make use of the notation

$$
\widetilde{\mathcal{D}} f(a)=\mathcal{D}\left(f \circ \varphi_{a}\right)(0) \text { and } \widetilde{\nabla} f(z)=\nabla\left(f \circ \varphi_{a}\right)(0), \quad a \in B,
$$

for $f \in C^{1}(B)$. It is easy to see that $|\widetilde{\nabla} f|^{2}=2|\widetilde{\mathcal{D}} f|^{2}+2|\widetilde{\mathcal{D}} \bar{f}|^{2}$, which equals $4|\widetilde{\mathcal{D}} f|^{2}$ when $f$ is real-valued and of $C^{1}(B)$. It is $\mathcal{M}$-invariant in the sense that $|\widetilde{\nabla}(f \circ \psi)|=|(\widetilde{\nabla} f) \circ \psi|$ for all $\psi \in \mathcal{M}$ and $f \in C^{1}(B)$. We will also make use of the tangential gradient $\nabla_{T}$, which satisfies

$$
\left|\nabla_{T} f\right|^{2}=|\nabla f|^{2}-2|R f|^{2}-2|R \bar{f}|^{2}
$$

for $f \in C^{1}(B)$, where $R f$ denotes the radial derivative of $f$ :

$$
R f(z)=\sum_{j=1}^{n} z_{j} D_{j} f(z), \quad z \in B
$$


2.3. The surface area measure on $S$ normalized to have total mass one will be denoted by $\sigma$. Complex-valued continuous functions $f$ on $B$ satisfying the invariant mean value property:

$$
\int_{S} f \circ \varphi_{a}(r \zeta) d \sigma(\zeta)=f(a)
$$

for all $a \in B$ and all $r$ sufficiently small are called $\mathcal{M}$-harmonic (on $B$ ). $\mathcal{M}$-harmonic functions are real-analytic ( $[\mathrm{R}$, Theorem 4.2.5]), and are equivalently characterized by $C^{2}(B)$ functions $f$ satisfying $\widetilde{\Delta} f=0$ ([R, Theorem 4.2.4]).

Let $\nu$ be the Lebesgue volume measure of $\mathbb{C}^{n}=\mathbb{R}^{2 n}$ normalized to be $\nu(B)=1$ and let $\tau$ be the $\mathcal{M}$-invariant volume measure of $B$ :

$$
d \tau(z)=\frac{d \nu(z)}{\left(1-|z|^{2}\right)^{n+1}} .
$$

For $\zeta \in S$ and $1<\alpha<\infty, D_{\alpha}(\zeta)$ denotes the Koranyi approach region defined by

$$
D_{\alpha}(\zeta)=\left\{z \in B:|1-\langle z, \zeta\rangle|<\frac{\alpha}{2}\left(1-|z|^{2}\right)\right\} .
$$

Note that $D_{\alpha}(U \zeta)=U D_{\alpha}(\zeta)$ for all unitary operators $U$ of $\mathbb{C}^{n}$.

2.4. For a subharmonic function $f$ on $B$, we let $\|f\|_{p}=\lim _{r \rightarrow 1} M_{p}(r, f)$, where

$$
M_{p}(r, f)=\left(\int_{S}|f(r \zeta)|^{p} d \sigma(\zeta)\right)^{1 / p} .
$$

The $\mathcal{M}$-harmonic Hardy space $\mathcal{H}^{p}=\mathcal{H}^{p}(B), 0<p<\infty$, is defined to consist of those $f \mathcal{M}$-harmonic in $B$ for which $\|f\|_{\mathcal{H}^{p}}=\|f\|_{p}<\infty$. The Hardy space $H^{p}(B), 0<p<\infty$, is a subspace of $\mathcal{H}^{p}$ consisting of holomorphic functions. The hyperbolic Hardy class $\varrho H^{p}=\varrho H^{p}(B)$ is defined to consist of $\phi: B \rightarrow B_{1}$ for which $\|\phi\|_{\varrho H^{p}}=\|\varrho(\phi)\|_{p}<\infty$, where

$$
\varrho(\phi)=\varrho(\phi, 0)=\frac{1}{2} \log \frac{1+|\phi|}{1-|\phi|} .
$$

The Bloch space $\mathcal{B}$ consists of holomorphic functions $f$ in $B_{1}$ for which

$$
\sup _{z \in B_{1}}\left|f^{\prime}(z)\right|\left(1-|z|^{2}\right)<\infty .
$$

This is a Banach space, if the norm $\|f\|_{\mathcal{B}}$ of $f \in \mathcal{B}$ is defined to be the sum of $|f(0)|$ and the left side of the above inequality. See [AB], $\mathrm{ABC}$, BBG], CRW], [D], [G], [R], [Sto1], [FS], Zy], [Zhu] for $H^{p}$ theory, [K2], [K4], [Y1], Y2] for $\varrho H^{p}$ and $[\mathrm{ACP}],[\mathrm{Zhu}]$ for Bloch space.

2.5. Throughout this paper, $\phi$ always denotes a holomorphic function from $B$ into $B_{1}$ and we set

$$
\lambda(\phi)=\lambda \circ \phi=\log \frac{1}{1-|\phi|^{2}}, \quad|\widetilde{\phi}|=\frac{|\widetilde{\nabla} \phi|}{1-|\phi|^{2}} .
$$

Other undefined notation and terminology in this paper will follow the books by W. Rudin [R] and M. Stoll [Sto1]. 


\section{The INVARIANT LAPLACIAN AND THE INVARIANT GRADIENT}

Elementary properties involving $\widetilde{\Delta}$ and $\widetilde{\nabla}$ are prepared in this section.

The following representation of $|\widetilde{\nabla} f|$ is almost direct and so we omit its proof:

3.1. Proposition. Let $\Omega$ be an open subset of $B$. Then for $f \in C^{2}(\Omega)$ and $a \in \Omega$ the following equalities hold:

$$
\begin{gathered}
|\widetilde{\nabla} f(a)|^{2}=\lim _{r \rightarrow 0} \frac{2 n}{r^{2}} \int_{S}\left|f \circ \varphi_{a}(r \zeta)-f(a)\right|^{2} d \sigma(\zeta) \\
|\widetilde{\nabla} f(a)|^{2}=\lim _{r \rightarrow 0} \frac{2(n+1)}{r^{2(n+1)}} \int_{r B}\left|f \circ \varphi_{a}(w)-f(a)\right|^{2} d \tau(w) ; \\
|\widetilde{\nabla} f(a)|^{2}=2\left(1-|a|^{2}\right) \sum_{j, k=1}^{n}\left(\delta_{j, k}-a_{j} \bar{a}_{k}\right)\left\{D_{j} f \bar{D}_{k} \bar{f}+D_{j} \bar{f} \bar{D}_{k} f\right\}(a) ; \\
|\widetilde{\nabla} f(a)|^{2}=\left(1-|a|^{2}\right)\left|\nabla_{T} f(a)\right|^{2} \\
|\widetilde{\nabla} f(a)|^{2}=\frac{1}{2} \widetilde{\Delta}|f(a)|^{2}-\operatorname{Re}(\bar{f}(a) \widetilde{\Delta} f(a)) .
\end{gathered}
$$

3.2. Lemma. If $u$ and $v$ are real-valued and of $C^{2}(B)$, then

$$
\widetilde{\Delta}(u v)=u \widetilde{\Delta} v+v \widetilde{\Delta} u+2 \widetilde{\nabla} u \cdot \widetilde{\nabla} v .
$$

Proof. For $u, v \in C^{2}(B)$, elementary calculation gives

$$
\widetilde{\Delta}(u v)=u \widetilde{\Delta} v+v \widetilde{\Delta} u+4\langle\widetilde{\mathcal{D}} u, \widetilde{\mathcal{D}} \bar{v}\rangle+4\langle\widetilde{\mathcal{D}} v, \widetilde{\mathcal{D}} \bar{u}\rangle .
$$

Identifying $z=\left(z_{1}, \ldots, z_{n}\right) \in \mathbb{C}^{n}$ with $\left(\operatorname{Re} z_{1}, \operatorname{Im} z_{1}, \ldots, \operatorname{Re} z_{n}, \operatorname{Im} z_{n}\right) \in \mathbb{R}^{2 n}$, it is easy to see that $\nabla f \cdot \nabla g=4 \mathcal{D} f \cdot \mathcal{D} g$ for all real-valued $f$ and $g$, where $\cdot$ denotes the dot product of $\mathbb{R}^{2 n}$. Thus, for $a \in B$,

$$
\begin{aligned}
\operatorname{Re}\left\langle\mathcal{D}\left(u \circ \varphi_{a}\right)(0), \mathcal{D}\left(v \circ \varphi_{a}\right)(0)\right\rangle & =\mathcal{D}\left(u \circ \varphi_{a}\right)(0) \cdot \mathcal{D}\left(v \circ \varphi_{a}\right)(0) \\
& =\frac{1}{4} \widetilde{\nabla} u(a) \cdot \widetilde{\nabla} v(a)
\end{aligned}
$$

provided $u$ and $v$ are real-valued.

3.3. Lemma. Let $\Omega$ be an open subset of $B$ and $0<p<\infty$. Then for $f \in C^{2}(\Omega)$ and at the points of $\Omega$ where $f$ is nonzero, we have

$$
\widetilde{\Delta}|f|^{p}=\left.\left.p(p-2)|f|^{p-4}|\widetilde{\mathcal{D}}| f\right|^{2}\right|^{2}+\frac{p}{2}|f|^{p-2} \widetilde{\Delta}|f|^{2},
$$

which equals

$$
\widetilde{\Delta}|f|^{p}=p(p-1)|f|^{p-2}|\widetilde{\nabla} f|^{2}+p|f|^{p-2} f \widetilde{\Delta} f
$$

if $f$ is real-valued. Moreover, if $\widetilde{\Delta} f=0$ and $1<p<\infty$, then $\widetilde{\Delta}|f|^{p} \geq 0$ and

$$
\widetilde{\Delta}|f|^{p} \sim C_{p}|f|^{p-2}|\widetilde{\nabla} f|^{2} .
$$


Proof. (3.4) and (3.5) follow by direct calculation. Note that $\left.|\widetilde{\mathcal{D}}| f(a)\right|^{2}|\leq| f|| \widetilde{\nabla} f \mid$. Thus, if $\widetilde{\Delta} f=0$, then $\widetilde{\Delta}|f|^{2}=2|\widetilde{\nabla} f|^{2}$ by $(3.2)$; so

$$
p(p-1)|f|^{p-2}|\widetilde{\nabla} f|^{2} \leq \widetilde{\Delta}|f|^{p} \leq p|f|^{p-2}|\widetilde{\nabla} f|^{2}, \quad \text { if } 1<p \leq 2,
$$

and

$$
p|f|^{p-2}|\widetilde{\nabla} f|^{2} \leq \widetilde{\Delta}|f|^{p} \leq p(p-1)|f|^{p-2}|\widetilde{\nabla} f|^{2}, \quad \text { if } 2 \leq p<\infty,
$$

off zeros of $f$. This gives (3.6).

3.4. Let $0<r \leq 1$ and let

$$
G_{r}(z)=G(r, z)=\frac{1}{2 n} \int_{|z|}^{r} \frac{\left(1-t^{2}\right)^{n-1}}{t^{2 n-1}} d t, \quad z \in r B,
$$

and $G(z)=G_{1}(z)$. Then $\widetilde{\Delta} G(z)=0$ for all $z \in B, z \neq 0$. Thus $G$ is $\mathcal{M}$ superharmonic on $B \backslash\{0\}, G(0)=\infty$ and $\lim _{|z| \rightarrow 1} G(z)=0$. The function

$$
\mathbb{G}(z, w)=G\left(\varphi_{w}(z)\right), \quad z, w \in B,
$$

is called the (invariant) Green's function for $\widetilde{\Delta}$. It satisfies $\mathbb{G}(z, w)=\mathbb{G}(w, z)$ and $\widetilde{\Delta}_{z} \mathbb{G}(z, w)=0$ on $B \backslash\{0\}$. We recall the following Green's Theorem ( $[\mathrm{P}$, Lemma $2.5])$ :

$$
\int_{S} f(r \zeta) d \sigma(\zeta)-f(0)=\int_{r B} G(r, z) \widetilde{\Delta} f(z) d \tau(z)
$$

for $f \in C^{2}(B)$ and $0<r<1$.

3.5. Lemma. If $1<p<\infty$ and $f$ is (complex-valued) $\mathcal{M}$-harmonic on $B$, then

$$
\int_{S}|f(r \zeta)|^{p} d \sigma(\zeta)-|f(0)|^{p}=\int_{r B} G(r, z) \widetilde{\Delta}|f(z)|^{p} d \tau(z) .
$$

If $0<p<\infty$ and $\phi: B \rightarrow B_{1}$ is holomorphic, then

$$
\int_{S}(\lambda \circ \phi)^{p}(r \zeta) d \sigma(\zeta)-(\lambda \circ \phi)^{p}(0)=\int_{r B} G(r, z) \widetilde{\Delta}(\lambda \circ \phi)^{p}(z) d \tau(z) .
$$

Proof. In [Sto1], (3.8) is proved when $f$ is real-valued $\mathcal{M}$-harmonic on $B$. By applying a $C^{2}(B)$ function $f_{\epsilon}=f_{p, \epsilon}=\left(|f|^{2}+\epsilon\right)^{p / 2}, 0<\epsilon<1$, which is bounded if $2 \leq p<\infty$ and dominated by $C_{p}\left(|\widetilde{\Delta} \operatorname{Re} f|^{2}+|\widetilde{\Delta} \operatorname{Im} f|^{2}\right)$ if $1<p<2$, and using the limiting argument $\epsilon \rightarrow 1$ as in [Sto1, Proof of Theorem 6.18], we have (3.8). The same idea is applicable in proving (3.9) as follows.

Fix $p$ and $r: 0<p<\infty, 0<r<1$. Let

$$
\lambda_{\epsilon}(z)=\log \frac{1+\epsilon}{1-|z|^{2}}, \quad z \in B, 0<\epsilon<1 .
$$

Then $\left(\lambda_{\epsilon} \circ \phi\right)^{p} \in C^{2}(B),\left(\lambda_{\epsilon} \circ \phi\right)^{p} \downarrow(\lambda \circ \phi)^{p}$ as $\epsilon \downarrow 0$ monotonically, and

$$
\widetilde{\Delta}\left(\lambda_{\epsilon} \circ \phi\right)^{p}=p\left(\lambda_{\epsilon} \circ \phi\right)^{p-2}|\widetilde{\phi}|^{2}\left\{(p-1)|\phi|^{2}+\lambda_{\epsilon} \circ \phi\right\} .
$$

Also,

$$
\widetilde{\Delta}\left(\lambda_{\epsilon} \circ \phi\right)^{p} \geq 0, \quad \widetilde{\Delta}(\lambda \circ \phi)^{p} \geq 0
$$

and

$$
\widetilde{\Delta}\left(\lambda_{\epsilon} \circ \phi\right)^{p} \rightarrow(\lambda \circ \phi)^{p}
$$


pointwise almost everywhere in $B$.

If $1 \leq p<\infty$, then

$$
\widetilde{\Delta}\left(\lambda_{\epsilon} \circ \phi\right)^{p} \leq p^{2}|\widetilde{\phi}|^{2}\left(\lambda_{\epsilon} \circ \phi\right)^{p-1}
$$

because $|\phi|^{2} \leq \lambda_{\epsilon} \circ \phi$, so that $\left(\lambda_{\epsilon} \circ \phi\right)^{p}$ is uniformly bounded on $r B$. Since $G(r, z)$ is $\tau$ integrable on $r B$ and $\left(\lambda_{\epsilon} \circ \phi\right)^{p} \in C^{2}(B)$, we have

$$
\begin{aligned}
& \int_{S}(\lambda \circ \phi)^{p}(r \zeta) d \sigma(\zeta)-(\lambda \circ \phi)^{p}(0) \\
= & \lim _{\epsilon \rightarrow 0}\left\{\int_{S}\left(\lambda_{\epsilon} \circ \phi\right)^{p}(r \zeta) d \sigma(\zeta)-\left(\lambda_{\epsilon} \circ \phi\right)^{p}(0)\right\} \\
= & \lim _{\epsilon \rightarrow 0} \int_{r B} G(r, z) \widetilde{\Delta}\left(\lambda_{\epsilon} \circ \phi\right)^{p}(z) d \tau(z) \\
= & \int_{r B} \lim _{\epsilon \rightarrow 0} G(r, z) \widetilde{\Delta}\left(\lambda_{\epsilon} \circ \phi\right)^{p}(z) d \tau(z) \\
= & \int_{r B} G(r, z) \widetilde{\Delta}(\lambda \circ \phi)^{p}(z) d \tau(z) .
\end{aligned}
$$

This gives (3.9) for $1 \leq p<\infty$.

Next, if $0<p \leq 1$, then

$$
\widetilde{\Delta}\left(\lambda_{\epsilon} \circ \phi\right)^{p} \leq p|\widetilde{\phi}|^{2}\left(\lambda_{\epsilon} \circ \phi\right)^{p-1} \leq p|\widetilde{\phi}|^{2}(\lambda \circ \phi)^{p-1} \leq \frac{1}{p} \widetilde{\Delta}(\lambda \circ \phi)^{p} .
$$

By Fatou's lemma and Green's theorem,

$$
\begin{aligned}
& \int_{r B} G(r, z) \widetilde{\Delta}(\lambda \circ \phi)^{p}(z) d \tau(z) \\
= & \int_{r B} G(r, z) \liminf _{\epsilon \rightarrow 0} \widetilde{\Delta}\left(\lambda_{\epsilon} \circ \phi\right)^{p}(z) d \tau(z) \\
\leq & \liminf _{\epsilon \rightarrow 0} \int_{r B} G(r, z) \widetilde{\Delta}\left(\lambda_{\epsilon} \circ \phi\right)^{p}(z) d \tau(z) \\
= & \lim _{\epsilon \rightarrow 0}\left\{\int_{S}\left(\lambda_{\epsilon} \circ \phi\right)^{p}(r \zeta) d \sigma(\zeta)-\left(\lambda_{\epsilon} \circ \phi\right)^{p}(0)\right\} \\
= & \int_{S}(\lambda \circ \phi)^{p}(r \zeta) d \sigma(\zeta)-(\lambda \circ \phi)^{p}(0) .
\end{aligned}
$$

(3.11) and (3.12) show that $\widetilde{\Delta}\left(\lambda_{\epsilon} \circ \phi\right)^{p}$ is dominated by an $L^{1}(r B, G(r, \cdot) d \tau)$ function $p^{-1} \widetilde{\Delta}(\lambda \circ \phi)^{p}$. Thus, by the dominated convergence theorem, process $(3.10)$ is valid in this case also. This gives (3.9) for $0<p<1$.

For the case of real $\mathcal{H}^{p}$, the first half, (3.13) and (3.14) of the following theorem appeared in [Sto2].

3.6. Theorem. If $1<p<\infty$, then $f \in \mathcal{H}^{p}$ if and only if

$$
\int_{B}\left(1-|z|^{2}\right)^{n}|f(z)|^{p-2}|\widetilde{\nabla} f(z)|^{2} d \tau(z)<\infty .
$$

In this case,

$$
\|f\|_{p}^{p}=|f(0)|^{p}+\int_{B} G(z) \widetilde{\Delta}|f(z)|^{p} d \tau(z)
$$


If $0<p<\infty$ and $\phi: B \rightarrow B_{1}$ is holomorphic, then $\phi \in \varrho H^{p}(B)$ if and only if

$$
\int_{B}\left(1-|z|^{2}\right)^{n}(\lambda \circ \phi(z))^{p-1}|\widetilde{\phi}(z)|^{2} d \tau(z)<\infty .
$$

In this case,

$$
\|\lambda \circ \phi\|_{p}^{p}=|\lambda \circ \phi(0)|^{p}+\int_{B} G(z) \widetilde{\Delta}(\lambda \circ \phi)^{p}(z) d \tau(z) .
$$

Proof. Letting $r \rightarrow 1$, the monotone convergence theorem gives (3.14) and (3.16) from (3.8) and (3.9) respectively. Noting that

$$
G(z)=\log \frac{1}{|z|}, \text { if } n=1, \quad G(z) \sim \frac{\left(1-|z|^{2}\right)^{n}}{|z|^{2 n-2}}, \text { if } n \geq 2
$$

(which follows easily via L'Hospital's rule), (3.13) follows from (3.6) and (3.14). By (3.6), (3.19) follows. Since

$$
\begin{gathered}
p(\lambda \circ \phi)^{p-1}|\widetilde{\phi}|^{2} \leq \widetilde{\Delta}(\lambda \circ \phi)^{p} \leq p^{2}(\lambda \circ \phi)^{p-1}|\widetilde{\phi}|^{2} \quad \text { if } \quad 1 \leq p<\infty, \\
p^{2}(\lambda \circ \phi)^{p-1}|\widetilde{\phi}|^{2} \leq \widetilde{\Delta}(\lambda \circ \phi)^{p} \leq p(\lambda \circ \phi)^{p-1}|\widetilde{\phi}|^{2} \quad \text { if } \quad 0<p \leq 1,
\end{gathered}
$$

(3.15) follows from (3.16) and (3.17).

\section{Area function, $g$-Function and the maximal function}

In this section, we introduce a well-known characterization of $\mathcal{M}$-harmonic Hardy space in terms of the maximal function, the invariant area function, and the invariant $g$-function.

For $u \in C^{1}(B)$ and $1<\alpha<\infty$, we let

$$
\begin{gathered}
M_{\alpha} u(\zeta):=\sup \left\{|u(z)| ; z \in D_{\alpha}(\zeta)\right\}, \quad \zeta \in S ; \\
\widetilde{g}_{u}(\zeta):=\left(\int_{0}^{1} \frac{|\widetilde{\nabla} u(r \zeta)|^{2}}{1-r} d r\right)^{1 / 2}, \quad \zeta \in S ; \\
\widetilde{A}_{\alpha} u(\zeta):=\left(\int_{D_{\alpha}(\zeta)}|\widetilde{\nabla} u(z)|^{2} d \tau(z)\right)^{1 / 2}, \quad \zeta \in S .
\end{gathered}
$$

$\widetilde{A}_{\alpha}$ and $\widetilde{g}$ are $\mathcal{M}$-invariant in the sense that $\widetilde{g}_{u} \circ \psi=\widetilde{g}_{u \circ \psi}$ and $\widetilde{A}_{\alpha} u \circ \psi=\left(\widetilde{A}_{\alpha} u\right) \circ \psi$ for all $\psi \in \mathcal{M}$.

4.1. Theorem. For $\mathcal{M}$-harmonic $u$ and $1 \leq p<\infty$, the following four statements are equivalent:

$u=P[f]$ for some $f \in L^{p}(S) ; \quad M_{\alpha} u \in L^{p}(S) ; \quad \widetilde{A}_{\alpha} u \in L^{p}(S) ; \quad \widetilde{g}_{u} \in L^{p}(S)$, where $P[f]$ is the invariant Poisson integral of $f$ :

$$
P[f](z)=\int_{S} \frac{\left(1-|z|^{2}\right)^{n}}{|1-\langle z, \zeta\rangle|^{2 n}} f(\zeta) d \sigma(\zeta), \quad z \in B
$$

In ABC, Theorem 4.13], the first three statements of Theorem 4.1 are proved to be equivalent. So we prove the following to fulfill the proof of Theorem 4.1.

4.2. Theorem. For each $p: 1<p<\infty$,

$$
\|u\|_{\mathcal{H}^{p}} \sim\left\|\widetilde{g}_{u}\right\|_{L^{p}(S)}
$$

for all $u \in \mathcal{H}^{p}$ with $u(0)=0$. 
See $[\mathrm{Zy}]$ for a one-variable version of this result. See $[\mathrm{S}]$ also. We will prove Theorem 4.2 by a sequence of lemmas, which will also be helpful in proving our main result in Section 5 .

For $z \in B$ and $0<\delta \leq 1$, let us denote, as in [Sto1], $E(z, \delta)=\varphi_{z}(\delta B)$ in the sequel.

4.3. Lemma. For each $\alpha: 2<\alpha<\infty$, there is $\delta_{\alpha}: 0<\delta_{\alpha}<1$ such that

$$
E\left(r \zeta, \delta_{\alpha}\right) \subset D_{\alpha}(\zeta), \quad \zeta \in S,
$$

for all $r: 0<r<1$. For general $\alpha: 1<\alpha<\infty$, there are $\delta_{\alpha}: 0<\delta_{\alpha}<1$ and $r_{\alpha}: 0<r_{\alpha}<1$ such that (4.1) holds for all $r: r_{\alpha}<r<1$.

Proof. Let $w \in \delta B$ and let $z=\varphi_{r \zeta}(w)$. Then by $[\mathrm{R}$, Theorem $2.2 .5(2)]$ and by the fact that $\varphi_{r \zeta}(\zeta)=-\zeta$,

$$
\begin{aligned}
1-\langle z, \zeta\rangle & =1-\left\langle\varphi_{r \zeta}(w), \zeta\right\rangle \\
& =1-\left\langle\varphi_{r \zeta}(w), \varphi_{r \zeta} \circ \varphi_{r \zeta}(w)\right\rangle \\
& =\frac{\left(1-r^{2}\right)\left(1-\left\langle w, \varphi_{r \zeta}(\zeta)\right\rangle\right)}{(1-\langle w, r \zeta\rangle)\left(1-\left\langle r \zeta, \varphi_{r \zeta}(\zeta)\right\rangle\right)} \\
& =\frac{(1-r)(1+\langle w, \zeta\rangle)}{1-r\langle w, \zeta\rangle} .
\end{aligned}
$$

Thus,

$$
\begin{aligned}
|1-\langle z, \zeta\rangle| & =\left|\frac{(1-r)(1+\langle w, \zeta\rangle)}{1-r\langle w, \zeta\rangle}\right| \\
& =\frac{|(1+\langle w, \zeta\rangle)(1-r\langle w, \zeta\rangle)|}{(1+r)\left(1-|w|^{2}\right)}\left(1-|z|^{2}\right)
\end{aligned}
$$

which is at most

$$
\frac{1+r \delta}{(1-\delta)(1+r)}\left(1-|z|^{2}\right)
$$

Hence

$$
|1-\langle z, \zeta\rangle| \leq \frac{\alpha}{2}\left(1-|z|^{2}\right)
$$

when $\alpha>2$ by taking $\delta=\delta_{\alpha}=\frac{(\alpha-2)}{\alpha}$. This proves (4.1).

For general $\alpha: 1<\alpha<\infty$, by taking $\delta: \frac{\alpha-2}{\alpha}<\delta_{\alpha}<\frac{\alpha-1}{\alpha+1}$ and $r=r_{\alpha}$ that satisfy the equation $\frac{1+r \delta}{(1-\delta)(1+r)}=\frac{\alpha}{2}$, (4.1) also holds for all $r: r_{\alpha}<r<1$.

4.4. Lemma. For $0<p<\infty$ and for $\alpha: 1<\alpha<\infty, \widetilde{A}_{\alpha} u \in L^{p}(S)$ implies $\widetilde{g}_{u} \in L^{p}(S)$. In particular, for each $\alpha: 2<\alpha<\infty$, there is a positive constant $C_{\alpha}$ such that

$$
\widetilde{g}_{u}(\zeta) \leq C_{\alpha} \widetilde{A}_{\alpha} u(\zeta), \quad \zeta \in S
$$

for all $\mathcal{M}$ harmonic $u$.

Proof. Fix $\zeta \in S$. Note that if $w \in E(r \zeta, \delta)$, then $w=\varphi_{r \zeta}(z)$ for some $z:|z|<\delta$. So,

$$
1-|w|^{2}=\frac{\left(1-|z|^{2}\right)\left(1-r^{2}\right)}{|1-\langle z, r \zeta\rangle|^{2}} \leq\left(1-r^{2}\right)\left(\frac{1+|z|}{1-|z|}\right) \leq \frac{1+\delta}{1-\delta}\left(1-r^{2}\right)
$$


By the symmetry $w \in E(r \zeta, \delta) \Longleftrightarrow r \zeta \in E(w, \delta)$,

$$
\frac{1-\delta}{1+\delta}\left(1-r^{2}\right) \leq\left(1-|w|^{2}\right) \text {. }
$$

Thus,

$$
\delta_{1}(w):=\frac{-2 \delta+(1+\delta)|w|}{1-\delta}<r<\frac{2 \delta+(1-\delta)|w|}{1+\delta}:=\delta_{2}(w),
$$

whence it is not difficult to see that

$$
\begin{aligned}
& \int_{0}^{1}(1-r)^{-1} \mathcal{X}_{E(r \zeta, \delta)}(w) d r \\
\leq & \int_{\max \left\{\delta_{1}(w), 0\right\}}^{\delta_{2}(w)}(1-r)^{-1} d r \leq C_{\delta},
\end{aligned}
$$

where in the sequel $\mathcal{X}_{E}(w)=1$ if $w \in E$ and is 0 otherwise.

On the other hand, if $u$ is $\mathcal{M}$-harmonic, then

$$
|\widetilde{\nabla} u(r \zeta)|^{2} \leq C_{\delta} \int_{E(r \zeta, \delta)}|\widetilde{\nabla} u(w)|^{2} d \tau(w)
$$

(see [Sto1, Proposition 10.6]). Now, let $\delta=\delta_{\alpha}$ be that of (4.1). Then by (4.1) and (4.3),

$$
\begin{aligned}
\widetilde{g}_{u}(\zeta)^{2} & \leq C \int_{0}^{1}(1-r)^{-1} \int_{E(r \zeta, \delta)}|\widetilde{\nabla} u(w)|^{2} d \tau(w) d r \\
& \leq C \int_{D_{\alpha}(\zeta)}|\widetilde{\nabla} u(w)|^{2}\left(\int_{0}^{1}(1-r)^{-1} \mathcal{X}_{E(r \zeta, \delta)}(w) d r\right) d \tau(w) \\
& \leq C \int_{D_{\alpha}(\zeta)}|\widetilde{\nabla} u(w)|^{2} d \tau(w)=C \widetilde{A}_{\alpha} u(\zeta)^{2},
\end{aligned}
$$

where $C=C_{\delta}=C_{\alpha}$. This proves (4.2).

If $1<\alpha \leq 2$, then by the second part of Lemma 4.3,

$$
\widetilde{g}_{u}(\zeta)^{2} \leq \int_{0}^{r_{\alpha}}(1-r)^{-1}|\widetilde{\nabla} u(r \zeta)|^{2} d r+C_{\alpha} \widetilde{A}_{\alpha} u(\zeta)^{2},
$$

so that $\widetilde{A}_{\alpha} u \in L^{p}(S)$ implies $\widetilde{g}_{u} \in L^{p}(S)$.

4.5. Lemma. Let $1<p<\infty$ and $1<\alpha<\infty$. If $u \in \mathcal{H}^{p}$, then

$$
\int_{S} \int_{D_{\alpha}(\zeta)} \widetilde{\Delta}|u|^{p} d \tau d \sigma(\zeta) \leq C_{\alpha}\left(\int_{S}|u|^{p} d \sigma-|u|^{p}(0)\right) .
$$

Proof. Note that

$$
\begin{aligned}
& r \eta \in D_{\alpha}(\zeta) \Longleftrightarrow|1-\langle r \eta, \zeta\rangle|<\frac{\alpha}{2}\left(1-r^{2}\right) \\
\Longrightarrow & |1-\langle\eta, \zeta\rangle| \leq|1-\langle r \eta, \zeta\rangle|+(1-r) \leq(\alpha+1)(1-r) \\
\Longrightarrow & \zeta \in Q(\eta, \sqrt{(\alpha+1)(1-r)}),
\end{aligned}
$$

and since

$$
\sigma(Q(\eta, \sqrt{(\alpha+1)(1-r)})) \sim(1-r)^{n}
$$

([R] Proposition 5.1.4]), by (3.17) we have

$$
\int_{S} \mathcal{X}_{D_{\alpha}(\zeta)}(z) d \sigma(\zeta) \leq C_{\alpha}(1-|z|)^{n} \leq C_{\alpha} G(z) .
$$


Therefore it follows by (3.14) that

$$
\begin{aligned}
& \int_{S} \int_{D_{\alpha}(\zeta)} \widetilde{\Delta}|u|^{p} d \tau d \sigma(\zeta) \\
& \quad=\int_{B} \widetilde{\Delta}|u(z)|^{p}\left\{\int_{S} \mathcal{X}_{D_{\alpha}(\zeta)}(z) d \sigma(\zeta)\right\} d \tau(z) \\
& \quad \leq C_{\alpha} \int_{B} G(z) \widetilde{\Delta}|u(z)|^{p} d \tau(z) \\
& \quad \leq C_{\alpha}\left(\int_{S}|u|^{p} d \sigma-|u|^{p}(0)\right)
\end{aligned}
$$

4.6. Lemma. For each $p: 1<p<\infty$ and $\alpha: 1<\alpha<\infty$, there is a constant $C=C_{p, \alpha}$ such that

$$
\left\|\widetilde{A}_{\alpha} u\right\|_{L^{p}(S)} \leq C\|u\|_{\mathcal{H}^{p}}
$$

for all $u \in \mathcal{H}^{p}$.

Proof. Let $u \in \mathcal{H}^{p}$ and fix $\alpha: 1<\alpha<\infty$. Since

$$
\widetilde{\Delta} u=0 \Longleftrightarrow \widetilde{\Delta} \operatorname{Re} u=0=\widetilde{\Delta} \operatorname{Im} u,
$$

with the help of Minkowski's inequality we may assume in proving (4.7) that $u$ is real and nonconstant. Since $D_{\alpha} \subset D_{\beta}$ if $\alpha<\beta$, we also may assume that $2<\alpha<\infty$. We divide the proof into three cases with regard to the magnitude of $p$ and abbreviate $C_{p, \alpha}$ by $C$.

Case 1. First, suppose $1<p \leq 2$. Then, by Lemma 2 , on the set where $u$ is not zero,

$$
\widetilde{\Delta}|u|^{p}=p(p-1)|u|^{p-2}|\widetilde{\nabla} u|^{2}
$$

Since $u$ is real-analytic ( $[\mathbb{R}$, Theorem 4.2.5]), the zero set of $u$ is of volume measure zero. So,

$$
\begin{aligned}
\left(\widetilde{A}_{\alpha} u(\zeta)\right)^{p} & \leq C\left(\int_{D_{\alpha}(\zeta)}|u|^{2-p} \widetilde{\Delta}|u|^{p} d \tau\right)^{p / 2} \\
& \leq C\left(M_{\alpha} u(\zeta)\right)^{p(2-p) / 2}\left(\int_{D_{\alpha}(\zeta)} \widetilde{\Delta}|u|^{p} d \tau\right)^{p / 2} .
\end{aligned}
$$

Thus, Hölder's inequality, the complex maximal theorem ([R, Theorem 5.4.10]), and Lemma 4.5 give

$$
\begin{aligned}
\int_{S}\left(\widetilde{A}_{\alpha} u\right)^{p} d \sigma & \leq C \int_{S}\left(M_{\alpha} u(\zeta)\right)^{p(2-p) / 2}\left(\int_{D_{\alpha}(\zeta)} \widetilde{\Delta}|u|^{p} d \tau\right)^{p / 2} d \sigma(\zeta) \\
& \leq C\left(\int_{S}\left(M_{\alpha} u\right)^{p} d \sigma\right)^{(2-p) / 2}\left(\int_{S} \int_{D_{\alpha}(\zeta)} \widetilde{\Delta}|u|^{p} d \tau d \sigma(\zeta)\right)^{p / 2} \\
& \leq C\|u\|_{\mathcal{H}^{p}}^{p-\left(p^{2} / 2\right)}\left(\|u\|_{\mathcal{H}^{p}}^{p}-|u(0)|^{p}\right)^{p / 2} \\
& \leq C\|u\|_{\mathcal{H}^{p}}^{p}
\end{aligned}
$$

whence follows the desired inequality (4.7). 
Case 2. Next, we suppose $4 \leq p<\infty$. Let $h$ be a nonnegative polynomial with

$$
\|h\|_{L^{q}(S)} \leq 1, \quad \frac{2}{p}+\frac{1}{q}=1, \quad 1<q \leq 2,
$$

and let $H=P[h]: H(z)=\int_{S} P(z, \zeta) h(\zeta) d \sigma(\zeta)$.

Since

$$
\begin{aligned}
z \in D_{\alpha}(\zeta) & \Longleftrightarrow|1-\langle z, \zeta\rangle|<\frac{\alpha}{2}\left(1-|z|^{2}\right) \\
\Longrightarrow & \frac{1}{\left(1-|z|^{2}\right)^{n+1}}<\frac{1}{1-|z|^{2}}\left(\frac{\alpha}{2}\right)^{2 n} P(z, \zeta),
\end{aligned}
$$

it follows that

$$
\begin{aligned}
\widetilde{A}_{\alpha} u(\zeta) & =\left\{\int_{D_{\alpha}(\zeta)}|\widetilde{\nabla} u(z)|^{2} d \tau(z)\right\}^{1 / 2} \\
& \leq\left(\frac{\alpha}{2}\right)^{n}\left\{\int_{B}(1-|z|)^{-1} P(z, \zeta)|\widetilde{\nabla} u(z)|^{2} d \nu(z)\right\}^{1 / 2},
\end{aligned}
$$

whence by (3.17),

$$
\begin{aligned}
& \int_{S} h\left(\widetilde{A}_{\alpha} u\right)^{2} d \sigma \\
\leq & C \int_{S} h(\zeta)\left\{\int_{B}(1-|z|)^{-1} P(z, \zeta)|\widetilde{\nabla} u(z)|^{2} d \nu(z)\right\} d \sigma(\zeta) \\
= & C \int_{B}(1-|z|)^{-1} H(z)|\widetilde{\nabla} u(z)|^{2} d \nu(z) \\
\leq & C \int_{B} G(z)\left(H \widetilde{\Delta} u^{2}\right)(z) d \tau(z) .
\end{aligned}
$$

Since the last integrand is nonnegative,

$$
\int_{B} G(z)\left(H \widetilde{\Delta} u^{2}\right)(z) d \tau(z)=\lim _{r \rightarrow 1} \int_{r B} G(r, z)\left(H \widetilde{\Delta} u^{2}\right)(z) d \tau(z)
$$

by the monotonicity, and by (3.3),

$$
H \widetilde{\Delta} u^{2}=\widetilde{\Delta}\left(H u^{2}\right)-2 \widetilde{\nabla} H \cdot \widetilde{\nabla} u^{2},
$$

so that

$$
\begin{aligned}
& \int_{B} G(z)\left(H \widetilde{\Delta} u^{2}\right)(z) d \tau(z) \\
\leq & \left|\lim _{r \rightarrow 1} \int_{r B} G(r, \cdot) \widetilde{\Delta}\left(H u^{2}\right) d \tau\right|+2 \int_{B} G|\widetilde{\nabla} H|\left|\widetilde{\nabla} u^{2}\right| d \tau \\
= & (I)+(I I) .
\end{aligned}
$$

Now since $H$ is a bounded function and $u$ is dominated by the radial maximal function of $u$, an $L^{p}(S)$ function ([R Theorem 5.4.10]), (3.7) and the dominated convergence theorem give that

$$
\begin{aligned}
\lim _{r \rightarrow 1} \int_{r B} G(r, \cdot) \widetilde{\Delta}\left(H u^{2}\right) d \tau & =\lim _{r \rightarrow 1} \int_{S} H u^{2}(r \zeta) d \sigma-H u^{2}(0) \\
& =\int_{S} h u^{2}(\zeta) d \sigma-H u^{2}(0)
\end{aligned}
$$


Hence, by Hölder's inequality with pairing $\left(\frac{p}{2}, q\right)$,

$$
\begin{aligned}
(I) & =\left|\int_{S} h u^{2}(\zeta) d \sigma-H u^{2}(0)\right| \\
& \leq\left(\int_{S} h^{q} d \sigma\right)^{1 / q}\left(\int_{S}|u|^{p} d \sigma\right)^{2 / p} \\
& \leq\|u\|_{\mathcal{H}^{p}}^{2} .
\end{aligned}
$$

On the other hand, by a simple calculation, $\left|\widetilde{\nabla} u^{2}(z)\right|=2|u(z)||\widetilde{\nabla} u(z)|$. So by (4.2) and by Case 1 we just verified

$$
\left\|\widetilde{g}_{H}\right\|_{L^{q}} \leq C\left\|\widetilde{A}_{\alpha} H\right\|_{L^{q}} \leq C\|H\|_{L^{q}} \leq C\|h\|_{L^{q}} \leq C, \quad 1<q \leq 2,
$$

whence it follows by (3.17) that

$$
\begin{aligned}
& (I I) \leq 4 \int_{B} G|\widetilde{\nabla} H||u||\widetilde{\nabla} u| d \tau \\
& =4 \int_{0}^{1} \mathcal{G}(r) \int_{S}|u(r \zeta)||\widetilde{\nabla} H(r \zeta)||\widetilde{\nabla} u(r \zeta)| d \sigma(\zeta) d r \\
& \leq 4 \int_{S}\left(M_{\text {rad }} u\right)(\zeta)\left(\int_{0}^{1} \mathcal{G}(r)|\widetilde{\nabla} H(r \zeta)|^{2} d r\right)^{1 / 2} \\
& \quad \times\left(\int_{0}^{1} \mathcal{G}(r)|\widetilde{\nabla} u(r \zeta)|^{2} d r\right)^{1 / 2} d \sigma(\zeta) \\
& \leq C\left(\int_{S}\left(M_{\text {rad }} u\right)^{p} d \sigma\right)^{1 / p}\left(\int_{S} \widetilde{g}_{H}^{q} d \sigma\right)^{1 / q}\left(\int_{S} \widetilde{g}_{u}^{p} d \sigma\right)^{1 / p} \\
& \leq C\|u\|_{\mathcal{H}^{p}}\|H\|_{L^{q}}\left\|\widetilde{g}_{u}\right\|_{L^{p}} \\
& \leq C\|u\|_{\mathcal{H}^{p}}\left\|\widetilde{g}_{u}\right\|_{L^{p}},
\end{aligned}
$$

where $M_{\text {rad }} u$ denotes the radial maximal function of $u$ and

$$
\mathcal{G}(r)=2 n \frac{r^{2 n-1}}{\left(1-r^{2}\right)^{n+1}} G(r) .
$$

Gathering up from (4.10) to (4.13),

$$
\int_{S} h\left(\widetilde{A}_{\alpha} u\right)^{2} d \sigma \leq C\left(\|u\|_{\mathcal{H}^{p}}^{2}+\|u\|_{\mathcal{H}^{p}}\left\|\widetilde{g}_{u}\right\|_{L^{p}}\right)
$$

for all nonnegative polynomials $h$ with

$$
\|h\|_{L^{q}(S)} \leq 1, \quad \frac{2}{p}+\frac{1}{q}=1,1<q \leq 2 .
$$

Since polynomials (of $z_{j}$ and $\overline{z_{j}}, 1 \leq j \leq n$ ) are dense in $C(S)$ and $C(S)$ is dense in $L^{q}(S)$ in this case, we obtain

$$
\left\|\widetilde{A}_{\alpha} u\right\|_{L^{p}}^{2} \leq C\left(\|u\|_{\mathcal{H}^{p}}^{2}+\|u\|_{\mathcal{H}^{p}}\left\|\widetilde{g}_{u}\right\|_{L^{p}}\right)
$$

for all nonconstant real $u \in \mathcal{H}^{p}$. Now by using the arithmetic-geometric mean inequality we conclude (4.7) from (4.14). 
Case 3. It now remains to verify the lemma for $2<p<4$. Given $\alpha$, we define the operator $T_{\alpha}$ on $L^{2}(S)$ into the set of measurable functions as

$$
T_{\alpha} f(\zeta)=\left(\int_{D_{\alpha}(\zeta)} \widetilde{\Delta}|P[f]|^{2} d \tau\right)^{1 / 2} .
$$

Then $T_{\alpha}$ is subadditive by a simple application of Minkowski's inequality. If $u \in$ $\mathcal{H}^{p}(B), 2 \leq p \leq 4$, then $u=P[f]$ for some $f \in L^{p}(S)$ ([R, Theorem 4.3.3]), so that $\widetilde{A}_{\alpha} u=T_{\alpha} f$, whence (4.7) becomes

$$
\left\|T_{\alpha} f\right\|_{L^{p}} \leq C\|P[f]\|_{\mathcal{H}^{p}}=C\|f\|_{L^{p}},
$$

where the last equality comes from $[\mathrm{R}$ Theorem 5.4 .8 or Theorem 5.4.9], or [Sto1. Proposition 5.6]. But we already proved that (4.15) holds for $p=2$ and $p=4$ respectively in Case 1 and Case 2. This means that the operator $T_{\alpha}$ is simultaneously of type $(2,2)$ and of type $(4,4)$, whence, by the interpolation theorem of Marcinkiewicz $([\mathrm{Zy}])$, the operator is of type $(p, p), 2<p<4$. This in turn means that (4.15) holds for $2<p<4$, and gives that, with $P[f]=u$,

$$
\left\|\widetilde{A}_{\alpha} u\right\|_{L^{p}}=\left\|T_{\alpha} f\right\|_{L^{p}} \leq C\|f\|_{L^{p}}=C\|u\|_{L^{p}}=C\|u\|_{\mathcal{H}^{p}} .
$$

Now, by Lemma 4.4 and Lemma 4.6 we have

$$
\left\|\widetilde{g}_{u}\right\|_{L^{p}} \leq C_{p}\|u\|_{\mathcal{H}^{p}} .
$$

So to prove Theorem 4.2 it remains to show

4.7. Lemma. For each $p: 1<p<\infty$, there is a constant $C=C_{p}$ such that

$$
\|u\|_{\mathcal{H}^{p}} \leq C\left\|\widetilde{g}_{u}\right\|_{L^{p}}
$$

for all $u \in \mathcal{H}^{p}$ with $u(0)=0$.

Proof. With the help of Minkowski's inequality and a simple inequality: $A^{t}+B^{t} \leq$ $C(A+B)^{t}, 0<t<\infty$, we may assume $u$ to be real. If $f$ is a polynomial and $F=P[f]$, then by $(3.7)$,

$$
\int_{S} F u(r \zeta) d \sigma(\zeta)=\int_{r B} G(r, \cdot) \widetilde{\Delta}(F u) d \tau
$$

for all $r: 0<r<1$. Thus, by (3.3) and Hölder's inequality,

$$
\begin{aligned}
\left|\int_{S} F u(r \zeta) d \sigma(\zeta)\right| & \leq C \int_{r B} G(r, \cdot)|\widetilde{\nabla} F||\widetilde{\nabla} u| d \tau \\
& \leq C \int_{S} \int_{0}^{1}(1-r)^{-1}|\widetilde{\nabla} F(r \zeta)||\widetilde{\nabla} u(r \zeta)| d r d \sigma(\zeta) \\
& \leq C\left\|\widetilde{g}_{F}\right\|_{L^{p}}\left\|\widetilde{g}_{u}\right\|_{L^{q}},
\end{aligned}
$$

where $\frac{1}{p}+\frac{1}{q}=1$. But

$$
f(\zeta) u(\zeta)=\lim _{r \rightarrow 1} F(r \zeta) u(r \zeta)
$$

almost everywhere $(\underline{\mathrm{R}}$, Theorem 5.4.8]), $F$ is bounded and $u$ is dominated by the radial maximal function of $u(\underline{\mathrm{R}}$, Theorem 5.4.10]), so that the dominated convergence theorem guarantees that

$$
\lim _{r \rightarrow 1} \int_{S} F u(r \zeta) d \sigma(\zeta)=\int_{S} f u d \sigma .
$$


By (4.16)

Hence (4.18) gives

$$
\left\|\widetilde{g}_{F}\right\|_{L^{p}} \leq C\|F\|_{\mathcal{H}^{p}} \leq C\|f\|_{L^{p}}
$$

$$
\left|\int_{S} f u d \sigma\right| \leq C\|f\|_{L^{p}}\left\|\widetilde{g}_{u}\right\|_{L^{q}}
$$

for every polynomial $f$. Therefore we have

$$
\|u\|_{L^{q}}=\|u\|_{\mathcal{H}^{q}} \leq C\left\|\widetilde{g}_{u}\right\|_{L^{q}} .
$$

\section{Hyperbolic Hardy classes and Bloch to $H^{p}$ pullbacks}

As was expected from Section 1.2, we prove our main theorem, Theorem 5.1, in this section. It characterizes the membership of $\varrho H^{p}(B)$ in terms of the hyperbolic area function and the $g$-function. It also shows that the hyperbolic Hardy class characterizes the Bloch- $H^{p}(B)$ pullback problem: For which $\phi: B \rightarrow B_{1}$ does $g \circ \phi$ belong to $H^{p}(B)$ for all $g \in \mathcal{B}$ ? The problem was naturally called for as explained in Section 1.1.

For $\phi: B \rightarrow B_{1}$ holomorphic and $1<\alpha<\infty$, let us denote the hyperbolic version of the $g$-function, the area function and the maximal function as follows:

$$
\begin{gathered}
\varrho \widetilde{g}_{\phi}(\zeta)=\int_{0}^{1} \frac{|\widetilde{\phi}(r \zeta)|^{2}}{1-r} d r, \quad \zeta \in S ; \\
\varrho \widetilde{A}_{\alpha} \phi(\zeta)=\int_{D_{\alpha}(\zeta)}|\widetilde{\phi}(z)|^{2} d \tau(z), \quad \zeta \in S ; \\
\varrho M_{\alpha} \phi(\zeta)=\sup \left\{\log \frac{1}{1-|\phi(z)|^{2}}: z \in D_{\alpha}(\zeta)\right\}, \quad \zeta \in S .
\end{gathered}
$$

Recall that $\widetilde{\phi}=\frac{|\widetilde{\nabla} \phi|}{1-|\phi|^{2}}$. We pay attention to the difference of a half-power in the above definition when compared to the case of the $(\mathcal{M}$-harmonic) invariant $g$-function $\widetilde{g}_{f}$ and the area function $\widetilde{A}_{\alpha} f$ of Section 4 . The following is the main theorem of this paper.

5.1. Theorem. Let $0<p<\infty$ and $1<\alpha, \beta<\infty$. If $\phi: B \rightarrow B_{1}$ is holomorphic, then the following are equivalent:

$$
\begin{gathered}
\phi \in \varrho H^{p}(B) ; \\
\varrho M_{\beta} \phi(\zeta) \in L^{p}(S) ; \\
\varrho \widetilde{A}_{\alpha} \phi \in L^{p}(S) ; \\
\varrho \widetilde{g}_{\phi} \in L^{p}(S) ; \\
f \circ \phi \in H^{2 p}(B) \quad \text { for all } \quad f \in \mathcal{B}\left(B_{1}\right) .
\end{gathered}
$$

In the case $n=1$, the equivalences between (5.3), (5.4) and (5.5) have been obtained in PX, Theorem 1.1].

The proof of $(5.1) \Longrightarrow(5.2)$ follows from the following complex hyperbolic maximal theorem: 
5.2. Lemma. Let $0<p<\infty$ and $1<\alpha<\infty$. If $\phi: B \rightarrow B_{1}$ is holomorphic, then $\varrho M_{\alpha} \phi \in L^{p}(S)$ if and only if $\phi \in \varrho H^{p}(B)$. In fact,

$$
\int_{S}\left(\varrho M_{\alpha} \phi\right)^{p}(\zeta) d \sigma(\zeta) \leq C_{\alpha, p} \lim _{r \rightarrow 1} \int_{S}\left(\log \frac{1}{1-|\phi(r \zeta)|^{2}}\right)^{p} d \sigma(\zeta) .
$$

Proof. Let $u=\lambda \circ \phi=-\log \left(1-|\phi|^{2}\right)$. Note that $\varrho M_{\alpha} \phi(\zeta)=\sup \left\{u(z): z \in D_{\alpha}(\zeta)\right\}$ $=M_{\alpha} u(\zeta)$. Fix $r: 0<r<1$ for a moment. Since $u_{r}=\lambda \circ \phi_{r}$ is $\mathcal{M}$-subharmonic in $B$ and of $C(\bar{B})$,

$$
u_{r}(z) \leq P\left[u_{r}\right](z), \quad z \in B
$$

Thus,

$$
M_{\alpha} u_{r}(z) \leq M_{\alpha}\left(P\left[u_{r}\right]\right)(z), \quad z \in B .
$$

Denoting $M u_{r}$ the (Hardy-Littlewood) maximal function of $u_{r}$, by [ $\mathbb{R}$, Theorem 5.4.5] and $[\mathrm{R}$ Theorem 5.2.6] we therefore have

$$
\begin{aligned}
\int_{S}\left(M_{\alpha} u_{r}\right)^{p} d \sigma & \leq \int_{S}\left(M_{\alpha} P\left[u_{r}\right]\right)^{p} d \sigma \leq C_{\alpha, p} \int_{S}\left(M u_{r}\right)^{p} d \sigma \\
& \leq C_{\alpha, p} \int_{S}\left(u_{r}\right)^{p} d \sigma \leq C_{\alpha, p} \sup _{r} \int_{S} u^{p}(r \zeta) d \sigma(\zeta)
\end{aligned}
$$

for $1<p<\infty$. Letting $r \rightarrow 1$, we have the conclusion for $p>1$. For $0<p \leq 1$, applying the above process with an $\mathcal{M}$-subharmonic function $\left(\lambda \circ \phi_{r}\right)^{1 / n} \in L^{n p}$, $n p>1$, instead of $u_{r}=\lambda \circ \phi_{r}$, we have the same result.

In the process of our proof, we need some lemmas as in Section 4. First we will make use of a subharmonicity involved with $\widetilde{\phi}$ :

5.3. Lemma. Let $0<\delta<1$ and $E(a, \delta)=\varphi_{a}(\delta B), a \in B$. Let $\phi: B \rightarrow B_{1}$ be holomorphic. Then

$$
|\widetilde{\phi}(a)|^{2} \leq C_{\delta} \int_{E(a, \delta)}|\widetilde{\phi}(w)|^{2} d \tau(w) .
$$

Proof. Fix $a \in B$. For $f: B \rightarrow B_{1}$ holomorphic let $F=f \circ \varphi_{a}$ for a moment. For $r: 0<r<1$,

and

$$
D_{j}\left(F \circ \varphi_{r \zeta}\right)(0)=\sum_{k=1}^{m} D_{k} F(r \zeta) \frac{\partial\left(\varphi_{r \zeta}\right)_{k}}{\partial z_{j}}(0)
$$

$$
\frac{\partial\left(\varphi_{r \zeta}\right)_{k}}{\partial z_{j}}(0)=-s \delta_{j k}+\frac{s}{1+s} r^{2} \bar{\zeta}_{j} \zeta_{k}
$$

where $s=\sqrt{1-r^{2}}\left(\left[\underline{\mathbb{R}}\right.\right.$, p. 48]). Since $H_{k}(z):=(1-\langle z, a\rangle)^{2} D_{k} F(z)$ is holomorphic for each $k$, by considering the power series expansion of holomorphic functions and orthogonality of $\left\{\zeta^{\alpha}\right\}$ ([R, Proposition 1.4.8, Proposition 1.4.9])

$$
\begin{aligned}
& \int_{S}(1-\langle r \zeta, a\rangle)^{2} D_{j}\left(F \circ \varphi_{r \zeta}\right)(0) d \sigma(\zeta) \\
= & \sum_{k=1}^{n} \int_{S} H_{k}(r \zeta)\left\{-s \delta_{j k}+\frac{s}{1+s} r^{2} \bar{\zeta}_{j} \zeta_{k}\right\} d \sigma(\zeta) \\
= & H_{j}(0)\left\{-s+\frac{s}{1+s} r^{2} \int_{S} \bar{\zeta}_{j} \zeta_{j} d \sigma(\zeta)\right\} \\
= & D_{j} F(0)\left\{-s+\frac{s}{1+s} \frac{r^{2}}{n}\right\} .
\end{aligned}
$$


Since

it follows that

$$
1-r^{2}=s^{2}=s-\frac{s}{1+s} r^{2} \leq s-\frac{s}{1+s} \frac{r^{2}}{n}
$$

$$
\begin{aligned}
\left(1-r^{2}\right)\left|D_{j} F(0)\right| & \leq\left\{-s+\frac{s}{1+s} \frac{r^{2}}{n}\right\}\left|D_{j} F(0)\right| \\
& =\left|\int_{S}(1-\langle r \zeta, a\rangle)^{m} D_{j}\left(F \circ \varphi_{r \zeta}\right)(0) d \sigma(\zeta)\right|,
\end{aligned}
$$

from which it follows that

$$
\left(1-r^{2}\right)^{2}\left|D_{j} F(0)\right|^{2} \leq \int_{S}\left|D_{j}\left(F \circ \varphi_{r \zeta}\right)(0)\right|^{2}|1-\langle a, r \zeta\rangle|^{4} d \sigma(\zeta)
$$

for $j=1,2, \ldots, n$, whence

$$
\left(1-r^{2}\right)^{2}|\mathcal{D} F(0)|^{2} \leq \int_{S}|\widetilde{\mathcal{D}} F(r \zeta)|^{2}|1-\langle a, r \zeta\rangle|^{4} d \sigma(\zeta)
$$

Since $\widetilde{\Delta}|\phi|^{2 l+2}=\frac{(l+1)^{2}}{4}|\phi|^{2 l} \widetilde{\Delta}|\phi|^{2}$, by letting $f=\phi^{l+1}$, we have

$$
\left(1-r^{2}\right)^{2} \widetilde{\Delta}|\phi(a)|^{2}|\phi(a)|^{2 l} \leq \int_{S} \widetilde{\Delta}\left|\phi \circ \varphi_{a}(r \zeta)\right|^{2}\left|\phi \circ \varphi_{a}(r \zeta)\right|^{2 l}|1-\langle a, r \zeta\rangle|^{4} d \sigma(\zeta)
$$

for $l=0,1,2, \ldots$. Since $|f(a)|<1$, by using the geometric series expansion we have

$$
\left(1-r^{2}\right)^{2}|\widetilde{\phi}(a)|^{2} \leq \int_{S}\left|\tilde{\phi} \circ \varphi_{a}(r \zeta)\right|^{2}|1-\langle a, r \zeta\rangle|^{4} d \sigma(\zeta)
$$

which is equivalent to

$$
\frac{|\widetilde{\phi}(a)|^{2}}{\left(1-|a|^{2}\right)^{2}} \leq \int_{S} \frac{\left|\widetilde{\phi} \circ \varphi_{a}(r \zeta)\right|^{2}}{\left(1-\left|\varphi_{a}(r \zeta)\right|^{2}\right)^{2}} d \sigma(\zeta) .
$$

Now, integrating (5.8) with respect to $2 n r^{2 n-1}\left(1-r^{2}\right)^{-n-1} d r$ over the interval $(0, \delta)$ and noting that $1-\left|\phi_{a}(w)\right|^{2} \geq \frac{1-\delta^{2}}{4}\left(1-|a|^{2}\right)$ for all $w:|w|<\delta<1$, we obtain

$$
\begin{aligned}
\frac{|\widetilde{\phi}(a)|^{2}}{\left(1-|a|^{2}\right)^{2}} & \leq C_{\delta} \int_{\delta B} \frac{\left|\widetilde{\phi} \circ \varphi_{a}(w)\right|^{2}}{\left(1-\left|\varphi_{a}(w)\right|^{2}\right)^{2}} d \tau(w) \\
& \leq C_{\delta} \frac{1}{\left(1-|a|^{2}\right)^{2}} \int_{E(a, \delta)}|\widetilde{\phi}(w)|^{2} d \tau(w) .
\end{aligned}
$$

This completes the proof.

If we replace (4.4) by (5.7), then the proof of Lemma 4.4 still holds with $\varrho \widetilde{g}_{\phi}$ and $\varrho \widetilde{A}_{\alpha} \phi$ in place of $\widetilde{g}_{u}$ and $\widetilde{A}_{\alpha} u$ respectively. Hence, we have

5.4. Lemma. Let $\phi: B \rightarrow B_{1}$ be holomorphic. For $0<p<\infty$ and for $\alpha: 1<$ $\alpha<\infty, \varrho \widetilde{A}_{\alpha} \phi \in L^{p}(S)$ implies $\varrho \widetilde{g}_{\phi} \in L^{p}(S)$. In particular, for each $\alpha: 2<\alpha<\infty$, there is a positive constant $C_{\alpha}$ such that

$$
\varrho \widetilde{g}_{\phi}(\zeta) \leq C_{\alpha} \varrho \widetilde{A}_{\alpha} \phi(\zeta), \quad \zeta \in S .
$$

It is easy to see that the proof of Lemma 4.5 holds with $u=-\log \left(1-|\phi|^{2}\right)$ with $\phi \in \varrho H^{p}(B):$ 
5.5. Lemma. Let $0<p<\infty$ and $1<\alpha<\infty$. If $u=\lambda \circ \phi=-\log \left(1-|\phi|^{2}\right)$ with $\phi \in \varrho H^{p}(B)$, then

$$
\int_{S} \int_{D_{\alpha}(\zeta)} \widetilde{\Delta}|u|^{p} d \tau d \sigma(\zeta) \leq C_{\alpha}\left(\int_{S}|u|^{p} d \sigma-|u|^{p}(0)\right) .
$$

5.6. We now proceed with the proof that (5.2) implies (5.3). The proof below runs in a parallel line with the proof of Lemma 4.6, and here we make use of Theorem 4.2 , the main result of Section 4.

Proof of (5.2) $\Longrightarrow$ (5.3). We will show that for $0<p<\infty$ and $1<\alpha<\infty$, there is a constant $C=C_{\alpha, p}$ such that

$$
\left\|\varrho \widetilde{A}_{\alpha} \phi\right\|_{L^{p}} \leq C\|\varrho(\phi)\|_{p}
$$

for all $f \in \varrho H^{p}(B)$. It is sufficient to assume that $2<\alpha<\infty$. We divide into two cases.

Case 1. $0<p \leq 1$. Let $\phi \in \varrho H^{p}(B)$. Since $\lambda(\phi) \geq|\phi|^{2}$, it follows that

$$
\lambda(\phi)^{1-p} \widetilde{\Delta} \lambda(\phi)^{p}=p \lambda(\phi)^{1-p} \lambda(\phi)^{p-2}|\widetilde{\phi}|^{2}\left\{\lambda(\phi)+(p-1)|\phi|^{2}\right\} \geq p^{2}|\widetilde{\phi}|^{2}
$$

almost everywhere (off the zeros of $\phi$ ), where $\lambda(\phi)=-\log \left(1-|\phi|^{2}\right.$ ). So

$$
\begin{aligned}
& \int_{S}\left(\varrho \widetilde{A}_{\alpha} \phi\right)^{p} d \sigma=\int_{S}\left(\int_{D_{\alpha}(\zeta)}|\widetilde{\phi}(z)|^{2} d \tau(z)\right)^{p} d \sigma(\zeta) \\
\leq & \frac{1}{p^{2}} \int_{S}\left(\int_{D_{\alpha}(\zeta)}(\lambda \circ \phi)^{1-p}(z) \widetilde{\Delta}(\lambda \circ \phi)^{p}(z) d \tau(z)\right)^{p / 2} d \sigma(\zeta) \\
\leq & \frac{1}{p^{2}} \int_{S}\left(\varrho M_{\alpha} \phi\right)^{1-p}(\zeta)\left(\int_{D_{\alpha}(\zeta)} \widetilde{\Delta}(\lambda \circ \phi)^{p}(z) d \tau(z)\right)^{p / 2} d \sigma(\zeta) .
\end{aligned}
$$

Hölder's inequality gives

$$
\begin{aligned}
& \int_{S}\left(\varrho \widetilde{A}_{\alpha} \phi\right)^{p} d \sigma \\
\leq & \frac{1}{p^{2}}\left\{\int_{S}\left(\varrho M_{\alpha} \phi\right)^{p} d \sigma\right\}^{1-p}\left\{\int_{S} \int_{D_{\alpha}(\zeta)} \widetilde{\Delta}(\lambda \circ \phi)^{p}(z) d \tau(z) d \sigma(\zeta)\right\}^{p} .
\end{aligned}
$$

Hence by Lemma 5.2 and Lemma 5.5,

$$
\int_{S}\left(\varrho \widetilde{A}_{\alpha} \phi\right)^{p} d \sigma \leq C_{p}\|\lambda \circ \phi\|_{p}^{p} \leq C_{p}\|\varrho \circ \phi\|_{p}^{p}
$$

This gives (5.11).

Case 2. $1<p<\infty$. To prove (5.11) we consider $\int_{S} h\left(\varrho \widetilde{A}_{\alpha} \phi\right) d \sigma$ for all nonnegative polynomials $h$ with $\|h\|_{L^{q}} \leq 1, \frac{1}{p}+\frac{1}{q}=1$. Let $h$ be such a polynomial and $H=P[h]$.

By (4.8) it follows that

$$
\begin{aligned}
\varrho \widetilde{A}_{\alpha} \phi(\zeta) & =\int_{D_{\alpha}(\zeta)}|\widetilde{\phi}(z)|^{2} d \tau(z) \\
& \leq\left(\frac{\alpha}{2}\right)^{2 n} \int_{B}(1-|z|)^{-1} P(z, \zeta)|\widetilde{\phi}(z)|^{2} d \nu(z),
\end{aligned}
$$


whence by (3.17),

$$
\begin{aligned}
& \int_{S} h\left(\varrho \widetilde{A}_{\alpha} \phi\right) d \sigma \\
\leq & C \int_{S} h(\zeta)\left\{\int_{B}(1-|z|)^{-1} P(z, \zeta)|\widetilde{\phi}(z)|^{2} d \nu(z)\right\} d \sigma(\zeta) \\
\leq & C \int_{B}(1-|z|)^{-1} H(z) \widetilde{\Delta} u(z) d \nu(z) \\
\leq & C \int_{B} G(z)(H \widetilde{\Delta} u)(z) d \tau(z),
\end{aligned}
$$

where we let $\lambda \circ \phi=u$ for simplicity.

Since the last integrand is nonnegative,

$$
\int_{B} G(z)(H \widetilde{\Delta} u)(z) d \tau(z)=\lim _{r \rightarrow 1} \int_{r B} G(r, z)(H \widetilde{\Delta} u)(z) d \tau(z)
$$

by the monotonicity. Since $\widetilde{\Delta} H \equiv 0$, by $(3,3), H \widetilde{\Delta} u=\widetilde{\Delta}(H u)-2 \widetilde{\nabla} H \cdot \widetilde{\nabla} u$. Hence,

$$
\begin{aligned}
& \int_{B} G(z)(H \widetilde{\Delta} u)(z) d \tau(z) \\
\leq & \left|\lim _{r \rightarrow 1} \int_{r B} G(r, \cdot) \widetilde{\Delta}(H u) d \tau\right|+2 \int_{B} G|\widetilde{\nabla} H||\widetilde{\nabla} u| d \tau \\
= & (I)+(I I) .
\end{aligned}
$$

Now since $H u \in C^{2}(B)$, we can apply (3.7), and since $H$ is a bounded function and $u$ is dominated by its radial maximal function, an $L^{p}(S)$ function by the hypothesis, the dominated convergence theorem gives

$$
\begin{aligned}
\lim _{r \rightarrow 1} \int_{r B} G(r, \cdot) \widetilde{\Delta}(H u) d \tau & =\lim _{r \rightarrow 1} \int_{S} H(r \zeta) u(r \zeta) d \sigma(\zeta)-H(0) u(0) \\
& =\int_{S} h(\zeta) u(\zeta) d \sigma-H(0) u(0) .
\end{aligned}
$$

Hence by Hölder's inequality,

$$
\begin{aligned}
(I) & =\left|\int_{S} h(\zeta) u(\zeta) d \sigma(\zeta)-H(0) u(0)\right| \\
& \leq\|h\|_{L^{q}}\|u\|_{p} \leq\|u\|_{p} .
\end{aligned}
$$

On the other hand, simple calculations give

$$
|\widetilde{\nabla} u(z)|=|\widetilde{\nabla}(\lambda \circ \phi)(z)|=2|\phi(z)||\widetilde{\phi}(z)|
$$


and $|\phi| \leq \lambda(\phi)^{1 / 2}$, whence it follows that

$$
\begin{aligned}
(I I) & \leq 4 \int_{B} G|\phi||\widetilde{\nabla} H||\widetilde{\phi}| d \tau \\
& \leq C \int_{0}^{1} \frac{d r}{1-r} \int_{S} u^{1 / 2}(r \zeta)|\widetilde{\nabla} H(r \zeta)||\widetilde{\phi}(r \zeta)| d \sigma(\zeta) \\
& \leq C \int_{S} M_{r a d} u^{1 / 2}\left(\int_{0}^{1} \frac{|\widetilde{\nabla} H|^{2}}{1-r} d r\right)^{1 / 2}\left(\int_{0}^{1} \frac{|\widetilde{\phi}|^{2}}{1-r} d r\right)^{1 / 2} d \sigma \\
& \leq C\left(\int_{S} M_{r a d} u^{p} d \sigma\right)^{1 / 2 p}\left(\int_{S} g_{H}^{q} d \sigma\right)^{1 / q}\left(\int_{S}\left(\varrho \widetilde{g}_{\phi}\right)^{p} d \sigma\right)^{1 / 2 p}
\end{aligned}
$$

by an application of Hölder's inequality with indices $(2 p, 2 p, q)$. Therefore by (5.6) and Theorem 4.2, we obtain

$$
(I I) \leq C\|u\|_{p}^{1 / 2}\|H\|_{q}\left\|\varrho \widetilde{g}_{\phi}\right\|_{L^{p}}^{1 / 2} \leq C\|u\|_{p}^{1 / 2}\left\|\varrho \widetilde{g}_{\phi}\right\|_{L^{p}}^{1 / 2}
$$

Gathering up,

$$
\int_{S} h\left(\varrho \widetilde{A}_{\alpha} \phi\right) d \sigma \leq C\left(\|u\|_{p}+\|u\|_{p}^{1 / 2}\left\|\varrho \widetilde{g}_{\phi}\right\|_{L^{p}}^{1 / 2}\right)
$$

for all nonnegative polynomials $h$ with $\|h\|_{L^{q}(S)} \leq 1, \frac{1}{p}+\frac{1}{q}=1, \quad 1<q<\infty$. Therefore, by (5.9) and the density argument, we obtain

$$
\left\|\varrho \widetilde{A}_{\alpha} \phi\right\|_{L^{p}}^{2} \leq C\left(\|u\|_{p}+\|u\|_{p}^{1 / 2}\left\|\varrho \widetilde{A}_{\alpha} \phi\right\|_{L^{p}}^{1 / 2}\right) .
$$

Now by use of the arithmetic-geometric mean inequality we conclude that

$$
\left\|\varrho \widetilde{A}_{\alpha} \phi\right\|_{L^{p}}^{2} \leq C\|u\|_{p}=C\|\lambda \circ \phi\|_{p}
$$

provided $\phi \in \varrho H^{p}(B)$, where $C=C_{p, \alpha}$. This gives (5.11).

5.7. Proof of $(5.3) \Longrightarrow$ (5.4) follows from Lemma 5.4.

5.8. Proof of $(5.4) \Longrightarrow$ (5.5): Suppose (5.4). If $1<p<\infty$, then by Theorem 4.1 it suffices to show that $\widetilde{g}_{f \circ \phi} \in L^{2 p}(S)$ for all $f \in \mathcal{B}\left(B_{1}\right)$. But, $\left\|\varrho \widetilde{g}_{f \circ \phi}\right\|_{L^{2 p}}$ is easily seen to be bounded by $\|f\|_{\mathcal{B}}\left\|\varrho \widetilde{g}_{\phi}\right\|_{L^{p}}^{1 / 2}$. If $0<p \leq 1$, then by [AB] (or [KL]) it suffices to show that

$$
\int_{S}\left(\int_{0}^{1}(1-r)|\nabla(f \circ \phi)(r \zeta)|^{2} d r\right)^{p} d \sigma(\zeta)<\infty
$$

for all $f \in \mathcal{B}\left(B_{1}\right)$. Using the inequality $|\widetilde{\nabla} \phi(r \zeta)| \geq 2\left(1-r^{2}\right)|\nabla \phi(r \zeta)|$ (which is valid when $\phi$ is holomorphic), the left-hand side integral is bounded by a constant times $\|f\|_{\mathcal{B}}^{2 p}\left\|\varrho \widetilde{g}_{\phi}\right\|_{L^{p}}^{p}$.

5.9. Proof of $(5.5) \Longrightarrow$ (5.1): Suppose (5.5). Then by the closed graph theorem, the composition operator $\mathcal{C}_{\phi}$ maps $\mathcal{B}\left(B_{1}\right)$ boundedly into $H^{2 p}(B)$. For each nonnegative integer $k$, let $\gamma_{k}$ be the Rademacher functions (see [D] or $\left.\mathrm{Zy}\right]$ ):

$$
\gamma_{k}(t)=\operatorname{sign} \sin \left(2^{k+1} \pi t\right), \quad t \in[0,1] .
$$


Consider for each non-dyadic $t \in[0,1]$ the function

$$
f_{t}(z)=\sum_{0}^{\infty} \gamma_{k}(t) z^{2^{k}}, \quad z \in B_{1} .
$$

Noting that

$$
(1-|z|)\left|f_{t}^{\prime}(z)\right|<(1-|z|) \sum_{0}^{\infty} 2^{k}|z|^{2^{k}-1} \leq 2(1-|z|) \sum_{0}^{\infty}|z|^{k} \leq 2,
$$

it follows that $f_{t} \in \mathcal{B},\left\|f_{t}\right\|_{\mathcal{B}} \leq 4$. So by the assumption,

$$
\left\|f_{t} \circ \phi\right\|_{H^{2 p}}=\left\|\mathcal{C}_{\phi} f_{t}\right\|_{H^{2 p}} \leq C\left\|f_{t}\right\|_{\mathcal{B}} \leq C
$$

for all non-dyadic $t \in[0,1]$ and so for almost every $t \in[0,1]$.

On integrating the $2 p$ power of each side of the above inequality with respect to $t$, we have by the monotone convergence theorem,

$$
C>\lim _{r \rightarrow 1} \int_{0}^{1} \int_{S}\left|f_{t} \circ \phi(r \zeta)\right|^{2 p} d \sigma(\zeta) d t .
$$

By changing the order of the integration, the last integral equals

$$
\int_{S} \int_{0}^{1}\left|\sum_{0}^{\infty} \gamma_{k}(t) \phi(r \zeta)^{2^{k}}\right|^{2 p} d t d \sigma(\zeta) .
$$

Thus, by [Zy Theorem V-8.4]), we have

$$
C>\lim _{r \rightarrow 1} \int_{S}\left(\sum_{0}^{\infty}\left|\phi(r \zeta)^{2}\right|^{2^{k}}\right)^{p} d \sigma(\zeta) .
$$

On the other hand, it is quite elementary to see that

$$
\sum_{k=0}^{\infty}\left|\phi(r \zeta)^{2}\right|^{2^{k}} \geq \sum_{k=1}^{\infty} \frac{\left|\phi(r \zeta)^{2}\right|^{k}}{k}=\log \frac{1}{1-|\phi(r \zeta)|^{2}}
$$

Therefore, we have

$$
\infty>C>\lim _{r \rightarrow 1} \int_{S} \log \left(\frac{1}{1-|\phi(r \zeta)|^{2}}\right)^{p / 2} d \sigma(\zeta)
$$

This gives (5.1).

We close this section by showing that the boundedness of our $\mathcal{C}_{\phi}$ is equivalent to its compactness. See [Zha] for the result when $n=1$ and $1 \leq p<\infty$. Also, recently in $\mathrm{PX}$, Theorem 2.3] the result has been established for the case $n=1$ and $0<p<\infty$.

5.10. Theorem. The composition operator $\mathcal{C}_{\phi}: \mathcal{B} \rightarrow H^{p}$ is bounded if and only if $\mathcal{C}_{\phi}$ is compact.

Proof. Suppose that $\mathcal{C}_{\phi}: \mathcal{B} \rightarrow H^{p}$ is bounded. Then $f \circ \phi \in H^{p}$ for all $f \in \mathcal{B}$ and $|\phi(\zeta)|:=\left|\lim _{n \rightarrow \infty} \phi(r \zeta)\right|$ exists and is less than 1 for almost every $\zeta \in S$.

Let $\left\{f_{n}\right\} \subset \mathcal{B}$ be such that $\left\|f_{n}\right\|_{\mathcal{B}} \leq 1$. We are going to show that $\left\{\mathcal{C}_{\phi} f_{n}\right\}$ has a convergent subsequence in $H^{p}(B)$. Since

$$
\left|f_{n}(z)\right| \leq\left|f_{n}(0)\right|+\left\|f_{n}\right\|_{\mathcal{B}} \log \frac{1}{1-|z|}, \quad z \in B_{1},
$$


$\left\{f_{n}\right\}$ forms a normal family, so that there is a subsequence of $\left\{f_{n}\right\}$ that converges uniformly on compact subsets of $B_{1}$ to a holomorphic function $f$. Passing to the subsequence, we may assume that the sequence $\left\{f_{n}\right\}$ itself converges to $f$. $f$ belongs to $\mathcal{B}$ with $\|f\|_{\mathcal{B}} \leq 1$ because

$$
\left|f^{\prime}(z)\right|\left(1-|z|^{2}\right)=\lim _{n \rightarrow \infty}\left|f_{n}^{\prime}(z)\right|\left(1-|z|^{2}\right) \leq 1 .
$$

On the other hand, by (5.5) $\Longrightarrow(5.4)$ of Theorem 5.1, $\varrho \widetilde{g}_{\phi} \in L^{p / 2}(S)$. In particular, $\varrho \widetilde{g}_{\phi}(\zeta)<\infty$ a.e. $\zeta \in S$. Since

$$
\left|\widetilde{\nabla}\left(\left(f_{n}-f\right) \circ \phi\right)\right| \leq\left\|f_{n}-f\right\|_{\mathcal{B}}|\widetilde{\phi}| \leq 2|\widetilde{\phi}|,
$$

the dominated convergence theorem gives $\lim \widetilde{g}_{\left(f_{n}-f\right) \circ \phi}(\zeta)=0$ a.e. $\zeta \in S$. Also, by (5.12), $\widetilde{g}_{\left(f_{n}-f\right) \circ \phi}$ is dominated by $2\left\{\varrho \widetilde{g}_{\phi}\right\}^{1 / 2}$, which is an $L^{p}(S)$ function. So, the dominated convergence theorem again gives

$$
\lim _{n \rightarrow \infty} \int_{S}\left\{\widetilde{g}_{\left(f_{n}-f\right) \circ \phi}(\zeta)\right\}^{p} d \sigma(\zeta)=0 .
$$

Using [KL, Theorem 5.1] when $0<p \leq 1$ and using Theorem 4.1 when $1<p<\infty$,

$$
\int_{S}\left|\left(f_{n}-f\right) \circ \phi(\zeta)-\left(f_{n}-f\right) \circ \phi(0)\right|^{p} d \sigma(\zeta) \leq C_{p} \int_{S}\left\{\widetilde{g}_{\left(f_{n}-f\right) \circ \phi}(\zeta)\right\}^{p} d \sigma(\zeta),
$$

whence

$$
\lim _{n \rightarrow \infty} \int_{S}\left|\left(f_{n}-f\right) \circ \phi(\zeta)\right|^{p} d \sigma(\zeta)=0
$$

by (5.13). Therefore, $\mathcal{C}_{\phi} f_{n} \rightarrow \mathcal{C}_{\phi} f$ in $H^{p}(B)$.

\section{More equivalent Results}

As shown in (3.1), the magnitude of the invariant gradient may be estimated only by the tangential gradient $\nabla_{T}$. It is a general principle that the behavior along the tangential direction is twice better than along the radial direction. So our case is as follows:

6.1. Theorem. For $\phi: B \rightarrow B_{1}$ holomorphic and $0<p<\infty, 1<\alpha<\infty$, each of the following are equivalent:

$$
\begin{gathered}
\phi \in \varrho H^{p}(B) ; \\
\int_{0}^{1} \frac{\left|\nabla_{T} \phi(r \zeta)\right|^{2}}{\left(1-|\phi(r \zeta)|^{2}\right)^{2}} d r \in L^{p}(S) ; \\
\int_{0}^{1}(1-r) \frac{|\nabla \phi(r \zeta)|^{2}}{\left(1-|\phi(r \zeta)|^{2}\right)^{2}} d r \in L^{p}(S) ; \\
\int_{0}^{1}(1-r) \frac{|R \phi(r \zeta)|^{2}}{\left(1-|\phi(r \zeta)|^{2}\right)^{2}} d r \in L^{p}(S) .
\end{gathered}
$$

Proof. By (3.1), (6.2) is the same as (5.4). So, by Theorem 5.1, (6.1) $\Longleftrightarrow(6.2)$ follows. 
To prove $(6.1) \Longleftrightarrow(6.3)$, we make use of the following well-known fact for holomorphic $f$ in $B$ :

$$
f \in H^{p}(B) \Longleftrightarrow\left(\int_{0}^{1}(1-r)|\nabla f(r \zeta)|^{2} d r\right)^{1 / 2} \in L^{p}(S) .
$$

See $\mathrm{AB}]$ or $[\mathrm{KL}]$ for example.

Suppose (6.1). Then by Theorem 5.1, $g \circ \phi \in H^{2 p}$ for all $g \in \mathcal{B}$. On the other hand, there are Bloch functions $g_{1}$ and $g_{2}$ such that

$$
\left|g_{1}^{\prime}(z)\right|+\left|g_{2}^{\prime}(z)\right| \geq \frac{1}{1-|z|^{2}}, \quad z \in B_{1}
$$

([RU, Proposition 5.4]). Thus,

$$
\left|g_{1}^{\prime} \circ \phi(z)\right|+\left|g_{2}^{\prime} \circ \phi(z)\right| \geq \frac{1}{1-|\phi(z)|^{2}}, \quad z \in B .
$$

But by (6.5),

$$
\int_{0}^{1}(1-r)\left|\nabla\left(g_{j} \circ \phi\right)(r \zeta)\right|^{2} d r \in L^{p}(S), \quad j=1,2 .
$$

Therefore, it follows that

$$
\begin{aligned}
& \int_{S}\left(\int_{0}^{1}(1-r) \frac{|\nabla \phi(r \zeta)|^{2}}{\left(1-|\phi(r \zeta)|^{2}\right)^{2}} d r\right)^{p} d \sigma(\zeta) \\
\leq & \int_{S}\left\{\int_{0}^{1}(1-r)\left(\sum_{j=1}^{2}\left|g_{j}^{\prime} \circ \phi\right||\nabla \phi|(r \zeta)\right)^{2} d r\right\}^{p} d \sigma(\zeta) \\
\leq & C_{p} \sum_{j=1}^{2} \int_{S}\left(\int_{0}^{1}(1-r)\left|\nabla\left(g_{j} \circ \phi\right)(r \zeta)\right|^{2} d r\right)^{p} d \sigma(\zeta) \\
< & \infty
\end{aligned}
$$

because $\left|\nabla\left(g_{j} \circ \phi\right)\right|=\left|g_{j}^{\prime} \circ \phi\right||\nabla \phi|$.

Conversely, suppose (6.3). Since

$$
|\nabla(g \circ \phi)| \leq|\nabla \phi|\left|g^{\prime} \circ \phi\right| \leq\|g\|_{\mathcal{B}} \frac{|\nabla \phi|}{1-|\phi|^{2}}
$$

for $g \in \mathcal{B}$, it follows that

$$
\int_{0}^{1}(1-r)|\nabla(g \circ \phi)(r \zeta)|^{2} d r \in L^{p}(S) .
$$

Therefore, by (6.5), $g \circ \phi \in H^{2 p}(B)$ for all $g \in \mathcal{B}$. This implies (6.1) by Theorem 5.1. $(6.1) \Longleftrightarrow(6.4)$ follows exactly in the same way as $(6.1) \Longleftrightarrow(6.3)$ just proven.

In the same way we directly get

6.2. Theorem. For $\phi: B \rightarrow B_{1}$ holomorphic and $0<p<\infty, 1<\alpha<\infty$, each of the following are equivalent to $\phi \in \varrho H^{p}(B)$ :

$$
\int_{D_{\alpha}(\zeta)}(1-r) \frac{\left|\nabla_{T} \phi(z)\right|^{2}}{\left(1-|\phi(z)|^{2}\right)^{2}} d \tau(z) \in L^{p}(S) ;
$$




$$
\begin{aligned}
& \int_{D_{\alpha}(\zeta)}(1-r)^{2} \frac{|\nabla \phi(z)|^{2}}{\left(1-|\phi(z)|^{2}\right)^{2}} d \tau(z) \in L^{p}(S) \\
& \int_{D_{\alpha}(\zeta)}(1-r)^{2} \frac{|R \phi(z)|^{2}}{\left(1-|\phi(z)|^{2}\right)^{2}} d \tau(z) \in L^{p}(S) .
\end{aligned}
$$

\section{Characterizing the boundedness of the Composition operators}

\section{ON BLOCH SPACE}

7.1. Let $Y$ be a complete holomorphic function space defined in terms of $|f|$ or $|\widetilde{\nabla} f|$. Let $\varrho(Y)$ be the hyperbolic counterpart of $Y$ in the sense that it consists of those bounded functions $\phi,|\phi|<1$, whose membership is defined via $\varrho(\phi, 0)$ (or $\lambda(\phi))$ and $\widetilde{\phi}$ in place of Euclidean distance $|f(z)|$ and $|\widetilde{\nabla} f|$ respectively that is used in the definition of the membership " $f \in Y$ ". Then there is a general phenomenon stating

$$
\mathcal{C}_{\phi} \text { maps } \mathcal{B} \text { boundedly into } Y \quad \Longleftrightarrow \quad \phi \in \varrho(Y) \text {. }
$$

Examples of classes $\varrho(Y)$ are $\varrho H^{p}\left(B_{1}\right), 1 \leq p<\infty, \varrho B M O A(B), \varrho \operatorname{Lip}_{\alpha}\left(B_{1}\right)$, and $\varrho \operatorname{Lip}_{\alpha}^{p}\left(B_{1}\right)$. By [K1], [K5], [Y4], and [KC], (7.1) is known to be true for these classes. Theorem 5.1 states that (7.1) is true with $Y=H^{p}(B), 0<p<\infty$, also.

7.2. An Example. Consider the function

$$
F(z)=\frac{z_{1}^{2}}{1-z_{2}^{2}}, \quad z=\left(z_{1}, z_{2}\right) \in B_{2} .
$$

The composition operator induced by $F$ was first considered in [CK], and the authors there proved quite complexly that $F$ takes Bloch functions to $H^{p}$ for all $p: 0<p<\infty$. In view of Theorem 5.1, this fact can be verified by showing that $F \in \bigcap \varrho H^{p}\left(B_{2}\right)$. Noting that $|F(\zeta)|=\left|\lim _{r \rightarrow 1} F(r \zeta)\right|<1$ almost every $\zeta \in S$, it is sufficient to prove that $\int_{S}\left(\log \frac{1}{1-|F(\zeta)|^{2}}\right)^{p} d \sigma(\zeta)<\infty$ for all $p$. But the following process is almost direct:

$$
\begin{aligned}
& \left\{\int_{S}\left(\log \frac{1}{1-|F(\zeta)|^{2}}\right)^{p} d \sigma(\zeta)\right\}^{1 / p} \leq\left\{\int_{S}\left(\sum_{k=1}^{\infty} \frac{|F|^{2 k}}{k}\right)^{p} d \sigma\right\}^{1 / p} \\
\leq & \sum_{k=1}^{\infty}\left\{\int_{S}\left(\frac{|F|^{2 k}}{k}\right)^{p} d \sigma\right\}^{1 / p}=\sum_{1}^{\infty} \frac{1}{k}\left\{\int_{B_{1}}\left(\frac{1-|w|^{2}}{\left|1-w^{2}\right|}\right)^{2 k p} d \nu_{1}\right\}^{1 / p} \\
\leq & C \sum_{1}^{\infty} \frac{1}{k}\left\{\int_{0}^{1}\left(1-r^{2}\right)^{2 k p}\left(1-r^{2}\right)^{1-k p} r d r\right\}^{1 / p} \\
\leq & C \sum_{1}^{\infty} \frac{1}{k}\left(\frac{1}{k p+2}\right)^{1 / p} \leq C \sum_{1}^{\infty} \frac{1}{k^{1+1 / p}}<\infty
\end{aligned}
$$

for all $p: p \geq 1$.

It was not known whether $F$ had the Bloch-BMO pullback property ([CK] $)$. Concerning this problem, it was mentioned in [CK] that previously known methods (used by P. Ahern and W. Rudin) do not work for this F. See Remark (a) and (b) of [CK]. We resolve this problem in the sequel to this paper, [K3]. 


\section{ACKNOWLEDGMENT}

The author would like to thank the referee for pointing out reference [PX] where Theorem 5.10 and part of Theorem 5.1 have been established providing $n=1$.

\section{REFERENCES}

[A] Patrick. R. Ahern, On the behavior near a torus of functions holomorphic in the ball, Pacific J. Math. 107 (1983), 267-278. MR 84i:32023

[AB] P. Ahern and J. Bruna, Maximal and area integral characterization of Hardy-Sobolov spaces in the unit ball of $\mathbb{C}^{n}$, Revista Mathematica Iberoamericana 4(1) (1988), 123-153. MR 90h:32011

[ABC] P. Ahern J. Bruna and C. Cascante, $H^{p}$-theory for generalized M-harmonic functions in the unit ball, Indiana Univ. Math. J. 45 (1996), 101-135. MR 98f:32008

[ACP] J. M. Anderson, J. Clunie and Ch. Pommerenke, On Bloch functions and normal functions, J. Reine Angew. Math. 270 (1974), 12-37. MR 50:13536

[AR1] P. Ahern and W. Rudin, Bloch functions, BMO, and boundary zeros, Indiana Univ. Math. J. 36 (1987), 131-148. MR 88d:42036

[AR2] Paley-type gap theorems for $H^{p}$-functions on the ball, Indiana Univ. Math. J. 37 (1988), 255-275. MR 89k:32007

[BBG] Aline Bonami, Joaquim Bruna and Sandrine Grellier, On Hardy, BMO and Lipschitz spaces of invariantly harmonic functions in the unit ball, Proc. London Math. Soc. 77 (1998), 665-696. MR 99g:31008

[C1] Boo Rim Choe, Cauchy integral equalities and applications, Trans. Amer. Math. Soc. 315 (1989), 337-352. MR 89m:32010

[C2] Composition property of holomorphic functions on the ball, Michigan Math. J. 36 (1989), 289-301. MR 90g:32005

[CC] Jun Soo Choa and Boo Rim Choe, Composition with a homogeneous polynomial, Bull. Korean Math. Soc. 29 (1992), 57-63. MR 94d:32008

[CK] J. S. Choa and H. O. Kim, Composition with a nonhomogeneous bounded holomorphic function on the ball, Canad. J. Math. 41 (1989), 870-881. MR 91a:32006

[CM] Carl C. Cowen and Barbara D. MacCluer, Composition operators on spaces of analytic functions, CRC Press, Boca Raton, FL, 1994. MR 97i:47056

[CRU] B. R. Choe, W. Ramey and D. Ullrich, Bloch -to-BMOA pullbacks on the disk, Proc. Amer. Math. Soc. 125 (1997), 2987-2996. MR 97m:47039

[CRW] R. Coifman, R. Rochberg and G. Weiss, Factorization theorems for Hardy spaces in several variables, Ann. of Math. 103 (1976), 611-635. MR 54:843

[D] Peter. L. Duren, Theory of $H^{p}$ functions, Academic Press, New York, 1970. MR 42:3552

[FS] C. Fefferman and E. Stein, $H^{p}$ spaces of several variables, Acta Math. 129 (1971), 137193. MR 56:6263

[G] John. B. Garnett, Bounded analytic functions, Academic Press, New York, 1981. MR 83g:30037

[H] Sigurdur Helgason, Groups and geometric analysis, Academic Press, New York, NY, 1984. MR 86c:22017

[K1] E. G. Kwon, Composition of Blochs with bounded analytic functions, Proc. Amer. Math. Soc. 124 (1996), 1473-1480. MR 96j:30052

[K2] Fractional integration and the hyperbolic derivative, Bull. Austral. Math. Soc. 38 (1988), 357-364. MR 90a:30096

[K3] Holomorphic functions of Bergman BMO in the ball, preprint.

[K4] Mean growth of the hyperbolic Hardy class functions, Mathematica Japonica 35 (1990), 451-460. MR 91e:30064

[K5] On analytic functions of Bergman BMO in the ball, Canad. Math. Bull. 42(1) (1999), 97-103. MR 2001a:32005

[KC] E. G. Kwon and J. S. Choa, On hyperbolic Hardy class functions and composition operators, Kor. J. Math. Sciences 124 (1997), 119-126.

[KL] Steven G. Krantz and Song-Ying Li, Area integral characterizations of functions in Hardy spaces on domains in $\mathbb{C}^{n}$, Complex Variables 32 (1997), 373-399. MR 98m:32003 
[Ma] Shamil Makhmutov, On Bloch-to-Besov composition operators, Proc. Japan. Acad. Ser. A Math. Sci. 72 (1996), 232-234. MR 98k:47058b

[P] M. Pavlović, Inequalities for the gradient of eigenfunctions of the invariant Laplacian in the unit ball, Indag. Math., N.S. 2(1) (1991), 89-98. MR 92d:32008

[PX] F. Pérez-González and J. Xiao, Bloch-Hardy pullbacks, preprint.

[R] Walter Rudin, Function theory in the unit ball of $\mathbb{C}^{n}$, Springer-Verlag, New York, 1980. MR 82i:32002

[RU] Wade Ramey and David Ullrich, Bounded mean oscillations of Bloch pull-backs, Math. Ann. 291 (1991), 591-606. MR 92i:32004

[Rus] P. Russo, Boundary behavior of BMOA(B $\left.B_{n}\right)$, Trans. Amer. Math. Soc. 292 (1985), 733740. MR 87d:32030

[S] E. M. Stein, Singular integrals and differentiability properties of functions, Princeton Univ. Press, 1970. MR 44:7280

[Sta] Charles S. Stanton, $H^{p}$ and BMOA pullback properties of smooth maps, Indiana Univ. Math. J. 40 (1991), 1251-1265. MR 93c:32006

[Sto1] M. Stoll, Invariant potential theory in the unit ball of $\mathbb{C}^{n}$, New York, 1994. MR 96f:31011

[Sto2] - A characterization of Hardy spaces of the unit ball of $\mathbb{C}^{n}$, J. London Math. Soc. 48 (1993), 126-136. MR 94g:32006

[Y1] Shinji Yamashita, Hyperbolic Hardy classes and hyperbolically Dirichlet-finite functions, Hokkaido Math. J., Special Issue 10 (1981), 709-722. MR 83i:30048

[Y2] _ Hyperbolic Hardy class $H^{1}$, Math. Scand. 45 (1979), 261- 266. MR 81i:30059

[Y3] Holomorphic functions of hyperbolically bounded mean oscillation, Boll. Un. Mat. Ital. B (6) 5 (1986), 983-1000. MR 88e:30092

[Y4] Smoothness of the boundary values of functions bounded and holomorphic in the disk, Trans. Amer. Math. Soc. 272 (1982), 539-544. MR 83i:30049

[Zha] Ruhan Zhao, Composition operators from Bloch type spaces to Hardy Besov spaces, J. Math. Anal. Appl. 233 (1999), 749-766. MR 2000a:47051

[Zhu] Kehe Zhu, Operator theory in function spaces, Marcel Dekker, New York, 1990. MR 92c: 47031

[Zy] A. Zygmund, Trigonometric series, Cambridge Univ. Press, London, 1959. MR 21:6498

Department of Mathematics Educhtion, Andong National University, Andong 760749, S. KoreA

E-mail address: egkwon@andong.ac.kr 